\title{
Meta-analysis of the clinical performance of commercial SARS-CoV-2 nucleic acid and antibody tests up to 22 August 2020
}

Ivo Van Walle ${ }^{1,2}$, Katrin Leitmeyer ${ }^{1}$, Eeva K Broberg ${ }^{1}$, the European COVID-19 microbiological laboratories group ${ }^{3}$

1. European Centre for Disease Prevention and Control, Stockholm, Sweden

2. Centre for Infectious Disease Control, National Institute for Public Health and the Environment, The Netherlands

3. The members of the European COVID-19 microbiological laboratories group are listed under Investigators

Correspondence: Ivo Van Walle (ivo.van.walle@rivm.nl)

Investigators: The investigators are listed at the end of the article.

Citation style for this article:

Van Walle Ivo, Leitmeyer Katrin, Broberg Eeva K, the European COVID-19 microbiological laboratories group. Meta-analysis of the clinical performance of commercial SARS-CoV-2 nucleic acid and antibody tests up to 22 August 2020. Euro Surveill. 2021;26(45):pii=2001675. https://doi.org/10.2807/1560-7917. ES.2021.26.45.2001675

Article submitted on 12 Sep 2020 / accepted on 16 Aug 2021 / published on 11 Nov 2021

Background: Reliable testing for SARS-CoV-2 is key for the management of the COVID-19 pandemic.

Aim: We estimate diagnostic accuracy for nucleic acid and antibody tests 5 months into the COVID-19 pandemic, and compare with manufacturer-reported accuracy. Methods: We reviewed the clinical performance of SARS-CoV-2 nucleic acid and antibody tests based on 93,757 test results from 151 published studies and 20,205 new test results from 12 countries in the European Union and European Economic Area (EU/ EEA). Results: Pooling the results and considering only results with $95 \%$ confidence interval width $\leqslant 5 \%$, we found four nucleic acid tests, including one pointof-care test and three antibody tests, with a clinical sensitivity $\geq 95 \%$ for at least one target population (hospitalised, mild or asymptomatic, or unknown). Nine nucleic acid tests and 25 antibody tests, 12 of them point-of-care tests, had a clinical specificity of $\geq 98 \%$. Three antibody tests achieved both thresholds. Evidence for nucleic acid point-of-care tests remains scarce at present, and sensitivity varied substantially. Study heterogeneity was low for eight of 14 sensitivity and 68 of 84 specificity results with confidence interval width $\leq 5 \%$, and lower for nucleic acid tests than antibody tests. Manufacturer-reported clinical performance was significantly higher than independently assessed in 11 of 32 and four of 34 cases, respectively, for sensitivity and specificity, indicating a need for improvement in this area. Conclusion: Continuous monitoring of clinical performance within more clearly defined target populations is needed.

\section{Introduction}

Testing is one of the central pillars of public health actions in epidemic and pandemic situations to allow timely identification, contact tracing and isolation of infectious cases to reduce the spread of infectious diseases. In addition, it allows estimating disease incidence, disease prevalence, and prevalence and duration of humoral immunity. Reliable testing for severe acute respiratory syndrome coronavirus 2 (SARS-CoV-2) and timely reporting of the data to public health authorities is therefore key for the management of the coronavirus disease (COVID-19) pandemic. This requires appropriate and sufficiently accurate diagnostic tests to identify individuals who are currently infected with SARS-CoV-2 as well as those who have been infected in the past. Timely access to testing, sufficient supply of testing materials, availability of tests and related reagents and consumables as well as highthroughput testing are pivotal in this context.

By August 2020, a large number of commercial tests for SARS-CoV-2 RNA detection (nucleic acid tests) were available, as well as serological tests for SARS-CoV-2specific antibodies. The various types of tests can be used for different purposes and many of these tests have the CE certificate for in vitro diagnostics (CE-IVD) that indicates compliance with the European IVD directive (98/79/EC) and can thus be marketed in the countries in the European Union and European Economic Area (EU/EEA). In addition, the United States (US) Food and Drug Administration has granted emergency use authorisations for many commercial tests in the US, and the World Health Organization (WHO) maintains an emergency use listing of commercial tests $[1,2]$. It is, however, important to note that CE certification is based on a self-declaration of the test manufacturer, including the claims on performance of the test. Independent information on the clinical performance of these tests in terms of sensitivity and specificity is still limited, and yet this is critical for proper interpretation of results.

For this reason, the European Centre for Disease Prevention and Control (ECDC) launched a continuous 


\section{FIGURE 1}

Selection of public studies on clinical performance of SARS-CoV-2 nucleic acid and antibody tests, up to 22 August $2020(\mathrm{n}=151)$

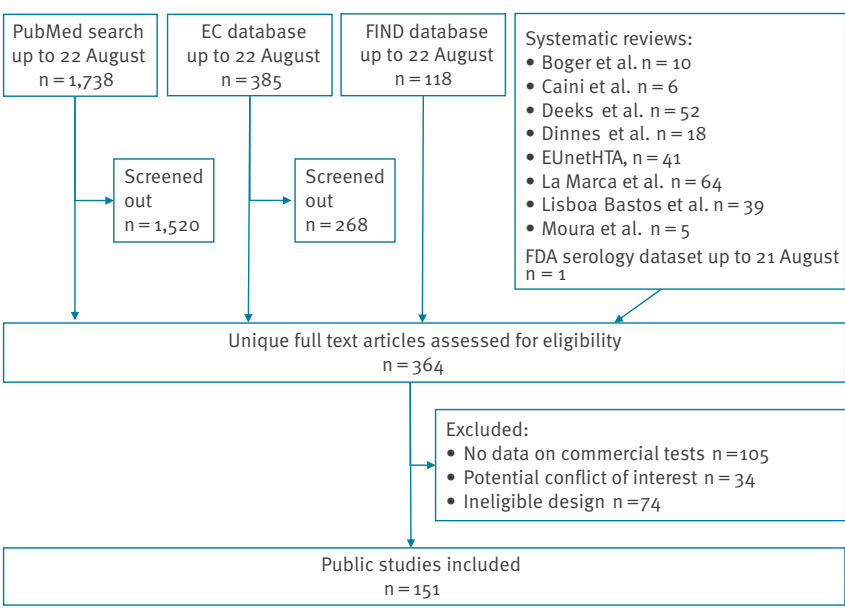

EC: European Commission COVID-19 In Vitro Diagnostic Devices and Test Methods database; FDA: United States Food and Drug Administration; FIND: Foundation for Innovative Diagnostics database; SARS-CoV-2: severe acute respiratory syndrome coronavirus 2 .

call to EU/EEA countries and the United Kingdom (UK) on 1 April 2020 to provide any such clinical performance data for sharing with other countries. These data, provided by 12 countries, are presented in this article. In addition, we included publicly available data. Finally, minimal performance criteria for different intended uses were gathered from public sources and aided by a survey conducted among EU/EEA countries and the UK from 20 May to 1 June 2020.

\section{Methods}

Search strategy and selection criteria

Studies containing potentially usable data on the clinical performance of SARS-CoV-2 nucleic acid and antibody tests were first extracted from systematic reviews on this topic. We identified these reviews through an initial PubMed (Medline) search for systematic reviews and meta-analyses for 'COVID-19' and 'SARS-CoV-2', followed by snowballing using the 'find similar articles' feature. We extended the selection with the studies listed in the Foundation for Innovative Diagnostics database (FIND, www.finddx.org/covid-19/tests) and the European Commission COVID-19 In Vitro Diagnostic Devices and Test Methods Database (EC, https://covid19-diagnostics.jrc.ec.europa.eu). Both databases attempt to exhaustively identify peer-reviewed as well as grey literature on clinical performance of COVID19 tests and are continuously updated [3,4]. Results from the latter were further filtered for articles with a description indicating that they contain clinical performance results. We also included results produced by the US Food and Drug Administration (FDA) [5]. Finally, we searched PubMed according to the query shown in Supplement 1.
The resulting studies were subsequently assessed for eligibility. By August 2020 there were no clinical performance studies that can be judged as having low risk of bias and low applicability concerns. Systematic reviews up to that point have not used risk of bias or applicability concerns as exclusion criteria [6-9]. This was not done in this work either. Instead, we excluded studies if they did not contain data on commercial tests, or if one or more of the authors were employed by the developer or manufacturer of the index test, to avoid possible conflicts of interest. Subsequently, we also excluded studies with an ineligible design, such as blinded tests, analytical validation only, use of another threshold for positivity than in the instructions for use, comparisons between different specimen types or use of an antibody rather than nucleic acid test as reference test for any type of index test.

Further exclusions were made at sample level based on the reference test employed. Samples classified as actual negatives, i.e. used for determining specificity, had to be taken (i) before the COVID-19 outbreak, in practice before 2020, (ii) from an individual without COVID-19-compatible symptoms, or (iii) from an individual with COVID-19-compatible symptoms but who was confirmed with another respiratory illness. Samples classified as actual negatives that were taken during the outbreak and were negative according to a nucleic acid test were therefore excluded. We did this to maximally reduce misclassification as actual negatives because of known issues with sensitivity of nucleic acid tests. Such misclassified samples would artificially lower index test specificity, in particular when the index test is more sensitive than the reference test [10-16]. For the same reason, the reported sensitivity of nucleic acid index tests, based on a nucleic acid reference test, was considered to be a positive agreement instead, calculated as part of a head-to-head comparison between the two tests. For antibody index tests on the other hand, we considered a nucleic acid test to be a valid reference test to determine actual positive samples and sensitivity, in accordance with WHO interim guidelines [17].

Manufacturer-reported clinical sensitivity and specificity data were extracted from instructions for use where available, or otherwise from the manufacturer's website. Sensitivity results derived from contrived samples spiked with purified viral RNA were excluded.

\section{Original clinical performance data}

Primary clinical performance data generated by the COVID-19 microbiological laboratories author group were assessed by the ECDC according to the same criteria as those of the literature review.

\section{Statistical analysis}

Meta-analysis of the included clinical sensitivity and specificity results was performed per test and per target, i.e. the genomic region for nucleic acid tests and the antibody isotype for antibody tests. Antibody test 
TABLE 1

Descriptive statistics on the number of published studies on clinical performance of SARS-CoV-2 nucleic acid and antibody tests, whether we included additional original data, and number of samples included in the meta-analysis, up to 22 August 2020 ( $\mathrm{n}=151$ studies $)$

\begin{tabular}{|c|c|c|c|c|c|c|c|c|}
\hline Country & Studies & $\begin{array}{c}\text { Original } \\
\text { data }\end{array}$ & $\begin{array}{c}\text { PCR } \\
\text { sens/spec }\end{array}$ & $\begin{array}{c}\text { CLIA } \\
\text { sens/spec }\end{array}$ & $\begin{array}{c}\text { ELISA } \\
\text { sens/spec }\end{array}$ & $\begin{array}{c}\text { LFIA } \\
\text { sens/spec }\end{array}$ & $\begin{array}{c}\text { Other } \\
\text { sens/spec }\end{array}$ & $\begin{array}{c}\text { Total } \\
\text { sens/spec }\end{array}$ \\
\hline Australia & 3 & No & $125 / 59$ & $0 / 0$ & $209 / 0$ & $1,511 / 1,012$ & $0 / 0$ & $1,845 / 1,071$ \\
\hline Austria & 5 & No & $115 / 75$ & $195 / 2,308$ & $421 / 0$ & $220 / 0$ & $0 / 0$ & $951 / 2,383$ \\
\hline Belgium & 6 & Yes & $22 / 6$ & $1,192 / 1,031$ & 957/922 & $3,934 / 2,985$ & $287 / 254$ & $6,392 / 5,198$ \\
\hline Brazil & 1 & No & $0 / 0$ & $0 / 0$ & o/o & $0 / 100$ & o/o & $0 / 100$ \\
\hline Canada & 1 & No & $0 / 0$ & $84 / 150$ & $185 / 150$ & $499 / 450$ & $0 / 0$ & $768 / 750$ \\
\hline China & 17 & No & $364 / 0$ & $3,659 / 1,572$ & $1,494 / 726$ & $1,038 / 557$ & $0 / 0$ & $6,555 / 2,855$ \\
\hline Croatia & 0 & Yes & $168 / 271$ & $0 / 0$ & $0 / 0$ & $0 / 0$ & $0 / 0$ & $168 / 271$ \\
\hline Cyprus & 0 & Yes & $6 / 466$ & $0 / 0$ & $0 / 0$ & $0 / 0$ & $0 / 0$ & $6 / 466$ \\
\hline Denmark & 2 & No & $0 / 0$ & $1,495 / 4,421$ & $195 / 1,403$ & $126 / 62$ & $0 / 0$ & $1,816 / 5,886$ \\
\hline Ecuador & 1 & No & $33 / 21$ & $0 / 0$ & $0 / 0$ & $0 / 0$ & $0 / 0$ & $33 / 21$ \\
\hline Finland & 3 & Yes & $121 / 75$ & $0 / 82$ & $64 / 238$ & $0 / 242$ & $0 / 0$ & $185 / 637$ \\
\hline France & 13 & Yes & $567 / 324$ & $173 / 165$ & $515 / 154$ & $1,160 / 486$ & $154 / 625$ & $2,569 / 1,754$ \\
\hline Germany & 9 & No & $85 / 200$ & $643 / 1,597$ & $508 / 568$ & $32 / 13$ & $0 / 0$ & $1,268 / 2,378$ \\
\hline Greece & 0 & Yes & $0 / 0$ & $0 / 0$ & $139 / 20$ & $0 / 0$ & $0 / 0$ & $139 / 20$ \\
\hline Hong Kong SAR & 1 & No & $72 / 114$ & $0 / 0$ & $0 / 0$ & $0 / 0$ & $0 / 0$ & $72 / 114$ \\
\hline Italy & 10 & No & $0 / 0$ & $139 / 37$ & $531 / 203$ & $60 / 97$ & $0 / 0$ & $730 / 337$ \\
\hline Japan & 5 & No & $340 / 435$ & $0 / 0$ & $0 / 0$ & $735 / 245$ & $98 / 111$ & $1,173 / 791$ \\
\hline Luxembourg & 0 & Yes & $0 / 0$ & $0 / 0$ & $235 / 218$ & $0 / 0$ & $0 / 0$ & $235 / 218$ \\
\hline The Netherlands & 4 & Yes & $253 / 210$ & $415 / 1,177$ & $2,107 / 3,449$ & $2,336 / 1,642$ & $0 / 0$ & $5,111 / 6,478$ \\
\hline Norway & 1 & No & $0 / 0$ & $0 / 0$ & $0 / 0$ & $207 / 0$ & $0 / 0$ & $207 / 0$ \\
\hline Poland & 0 & Yes & $390 / 662$ & $0 / 0$ & $0 / 0$ & $0 / 0$ & $0 / 0$ & $390 / 662$ \\
\hline Portugal & 0 & Yes & $0 / 0$ & $0 / 0$ & $0 / 0$ & $22 / 28$ & $0 / 0$ & $22 / 28$ \\
\hline Singapore & 2 & No & $0 / 0$ & $202 / 878$ & $0 / 0$ & $0 / 0$ & $0 / 0$ & $202 / 878$ \\
\hline Slovenia & 1 & Yes & $168 / 641$ & $0 / 0$ & $0 / 0$ & $0 / 0$ & $0 / 0$ & $168 / 641$ \\
\hline South Korea & 1 & No & $0 / 0$ & $0 / 0$ & $0 / 0$ & $140 / 158$ & $0 / 0$ & $140 / 158$ \\
\hline Spain & 4 & No & $0 / 0$ & $0 / 0$ & $0 / 124$ & $806 / 566$ & $0 / 0$ & $806 / 690$ \\
\hline Sweden & 2 & Yes & $39 / 4$ & $58 / 113$ & $0 / 0$ & $78 / 248$ & $0 / 0$ & $175 / 365$ \\
\hline Switzerland & 6 & No & $1,920 / 3,816$ & $0 / 0$ & $312 / 50$ & $129 / 50$ & $100 / 200$ & $2,461 / 4,116$ \\
\hline Taiwan & 1 & No & $0 / 0$ & $0 / 0$ & $0 / 0$ & $129 / 0$ & $0 / 0$ & $129 / 0$ \\
\hline United Kingdom & 17 & No & $15 / 1710$ & $1,975 / 5,247$ & $65 / 0$ & $412 / 200$ & $0 / 0$ & $2,467 / 7,157$ \\
\hline United States & 35 & No & $2,273 / 2,628$ & $1,260 / 4,164$ & $794 / 769$ & $5,446 / 11,140$ & $587 / 1,295$ & $10,360 / 19,996$ \\
\hline Total & 151 & $\mathrm{NA}$ & $7,076 / 11,717$ & $11,490 / 22,942$ & $8,731 / 8,994$ & $19,020 / 20,281$ & $1,226 / 2,485$ & $47,543 / 66,419$ \\
\hline
\end{tabular}

CLIA: chemiluminescence assay; ELISA: enzyme-linked immunosorbent assay; LFIA: lateral flow immunoassay; sens/spec: number of samples that are reference test positive/negative; NA: not applicable; SARS-CoV-2: severe acute respiratory syndrome coronavirus 2.

a Includes loop-mediated isothermal amplification, microarray, transcription-mediated amplification, and enzyme-linked fluorescent assay.

sensitivity results below the threshold number of days after onset were excluded. Sensitivity and positive agreement results were further stratified by case population as hospitalised cases, mild or asymptomatic cases, or unknown. We calculated pooled sensitivity and specificity values using fixed effects analysis, i.e. separately summing and dividing the number of correct predictions by the total number of samples in the group. Wilson score $95 \%$ confidence intervals (Cl) were calculated for pooled results. Study heterogeneity was assessed through the $\mathrm{I}^{2}$ statistic, calculated through random effects analysis using $\mathrm{R}$ version 4.0.2 and the metafor package [18]. We considered $\mathrm{I}^{2}$ values $<50.0 \%$ as low heterogeneity, $50.0-74.9 \%$ as moderate and $\geq 75 \%$ as high heterogeneity.

\section{Results}

\section{Minimum performance criteria}

By 1 June 2020, minimum performance criteria for tests were publicly available from Belgium, France, the Netherlands and the UK (Supplementary Table S1). All were applicable solely to antibody tests. The intended uses included diagnosis of COVID-19, determination of exposure to SARS-CoV-2 and determination of the immune status against SARS-CoV-2. Minimum clinical sensitivity for all of the specified intended uses ranged 
from $85 \%$ to $98 \%$, with a median of $95 \%$. These thresholds applied to samples collected at least 15 days post onset of symptoms (dpo), taking into account the time to seroconversion. Minimum clinical specificity for all of the specified intended uses was $98 \%$ in three countries and $98.5 \%$ in one. For nucleic acid confirmatory tests, the draft WHO Target Product Profiles for priority diagnostics to support response to the COVID-19 pandemic state >95\% to > $98 \%$ sensitivity (acceptable/ desired) and $>99 \%$ specificity [19].

We used general thresholds of $>95 \%$ sensitivity and $>98 \%$ specificity to determine if a test met the minimum performance criteria, together with a maximum $95 \% \mathrm{Cl}$ width $\leq 5 \%$. For results on IgM antibodies only, an upper limit of $\leq 28 \mathrm{dpo}$, or the highest dpo category with an upper limit $\leq 28 \mathrm{dpo}$, was added since IgM antibodies decrease fairly rapidly and such tests are not intended to be used long after exposure [20]. These sensitivity and specificity thresholds can be converted to false positives (FP) and negatives (FN), and positive and negative predictive value (PPV, NPV) if the prevalence of the condition, i.e. SARS-CoV-2 nucleic acid or antibody positivity, is known. These metrics better express the real impact of the accuracy. For a hypothetical low prevalence of $1 \%$ in a population of 100,000 people, the PPV would be $>32.4 \%$ $(\mathrm{FP}<1,980)$ and NPV > 99.9\% $(\mathrm{FN}<50)$. For a high prevalence of $5 \%$, these values would be $>71.4 \%$ (FP<1,900) and $>99.7 \%(\mathrm{FN}<250)$. Finally, for a high prevalence of $30 \%$, PPV would be $>95.3 \%(\mathrm{FP}<1,400)$ and NPV $>97.9 \%$ $(\mathrm{FN}<1,500)$.

\section{Primary clinical performance data}

We identified eight systematic reviews, including one by health technology assessment bodies not listed as a peer-reviewed study, and included the primary studies they were based on [6-9,21-24]. The full list of studies in the FIND and EC databases was retrieved on 22 August 2020. PubMed was searched on the same date. From the EC database, 268 of 385 studies were screened out because their description did not indicate that they contained clinical performance data on commercial tests. Of the remaining 117 studies, 81 were not present in the FIND database and 82 were not present in the EC database. From the PubMed results, 1,520 of 1,738 studies were screened out. From the combined list of 364 unique studies, 105 had no clinical performance data on commercial nucleic acid or antibody tests, 34 were excluded because of a potential conflict of interest and 74 were excluded because of ineligible design, leaving a total of 151 included studies. Of those, 53 were exclusively found through the Pubmed search and 15 in the FIND database. The remaining studies were listed by at least two sources.

A complete overview of the study selection is given in Figure 1. After exclusion of antibody test sensitivity results $\leq 14 \mathrm{dpo}$ and ineligible specificity results, a total of 37,435 and 56,322 index test results remained for calculation of sensitivity and specificity, respectively.
After addition of original, previously unpublished results provided by the authors of this study, this increased to 47,543 and 66,419 index test results, respectively, for 198 tests. A descriptive overview of the number of studies and results per country in given in Table 1. A complete overview of the studies is given in Supplementary Tables S2-S4.

\section{Meta-analysis}

Pooled estimates for clinical sensitivity and specificity per test, target and, for sensitivity, case population were made. For antibody tests, we restricted the results to those estimates that had a $95 \% \mathrm{Cl}$ width $\leq 5 \%$ and were derived from at least two studies, to be able to assess study heterogeneity. Based on the minimum performance criteria analysis, results $\geq 95 \%$ sensitivity and/or $\geq 98 \%$ specificity for a particular population are highlighted in Table 2. Among these results, there were two CLIA, one ELISA and no LFIA/POC that had $\geq 95 \%$ sensitivity and nine CLIA, four ELISAs and 12 LFIA/ POC that had $\geq 98 \%$ specificity, including the three with $\geq 95 \%$ sensitivity. Study heterogeneity was low for four of 10 sensitivity and 53 of 69 specificity results with $\mathrm{Cl}$ width $\leq 5 \%$. There were few sensitivity results for IgG for mild or asymptomatic cases, for IgA and for total antibody, none of which had a $\mathrm{Cl}$ width $\leq 5 \%$. In four cases where the same test was used for hospitalised cases, a reduction in sensitivity was observed of $7.4 \%, 11.0 \%, 13.1 \%$ and $19.2 \%$ for IgG (Table 2 ). For IgA and total antibody, data were available for only one test each. A reduction of $28.8 \%$ was observed for IgA and an increase of $6.0 \%$ for total antibody. The latter increase was probably due to the small number of samples for both populations.

For nucleic acid tests, results were restricted as for antibody tests (Table 3). Four tests, including one POC, had $\geq 95 \%$ positive agreement with a $\mathrm{Cl}$ width $\leq 5 \%$, and nine had $\geq 98 \%$ specificity. Study heterogeneity was low for all five sensitivity and all 15 specificity results with $\mathrm{Cl}$ width $\leq 5 \%$.

The correlation between independently assessed clinical performance results and manufacturer-reported results is shown in Figure 2. The manufacturer-reported documents are listed in Supplementary Table S2. Only independently assessed results with $\mathrm{Cl}$ width $\leq 5 \%$ are included. A total of 11 of 32 sensitivity and four of 33 specificity results reported by the manufacturer were significantly larger $(p<0.05)$.

\section{Discussion}

This review presents a comprehensive independent overview of clinical performance of commercially available nucleic acid and antibody tests 5 months into the COVID-19 pandemic. A substantial amount of previously unpublished data from European countries are included as well. By August 2020, there are numerous commercial tests for which sufficient performance data are available to allow calculation of clinical sensitivity or positive agreement, and specificity with narrow 
TABLE 2A

Pooled sensitivity and specificity results for SARS-CoV-2 antibody tests with confidence interval width $\leq 5 \%$ for either or both and based on at least two studies, up to 22 August 2020

\begin{tabular}{|c|c|c|c|c|c|}
\hline Category & Test & Target & Case population & Sensitivity $^{\mathrm{a}}$ & Specificity $^{a}$ \\
\hline CLIA & Abbott, SARS-CoV-2 IgG assay on Architect & IgG & Hospitalised & $\begin{array}{c}95.9(93.4-97.5) \\
n=368 \\
B E, C A, N L, U K, \text { US(3) }\end{array}$ & $\begin{array}{c}99.5(99.3-99.6) \\
n=8,243 \\
\text { AT, BE(2), CA, DE(2), DK, } \\
\text { FI, FR(3), IT, NL, SE, SG, } \\
\text { UK(3), US(8) }\end{array}$ \\
\hline CLIA & Abbott, SARS-CoV-2 IgG assay on Architect & IgG & Mild/asymptomatic & $\begin{array}{c}88.5(84.6-91.5)^{b} \\
n=331 \\
N L, \text { UK(2), US }\end{array}$ & Same as above \\
\hline CLIA & Abbott, SARS-CoV-2 IgG assay on Architect & $\lg G$ & Unk & $\begin{array}{c}92.0(90.4-93.3) \\
n=1,332 \\
\text { AT, BE, DE, DK, FI, } \\
\text { FR(2), SE, SG, UK(2), } \\
\text { US (4) }\end{array}$ & Same as above \\
\hline LFIA, POC & $\begin{array}{l}\text { Anhui Deep Blue Medical Technology, COVID-19 } \\
\text { (SARS-CoV-2) IgG/IgM Antibody Test Kit }\end{array}$ & IgG & $\mathrm{Na}$ & $\mathrm{Nd}$ & $\begin{array}{c}99.4(96.5-99.9) \\
n=158 \\
\text { CA, US }\end{array}$ \\
\hline ELISA & $\begin{array}{c}\text { Beijing Wantai Biological Pharmacy Enterprise, } \\
\text { Wantai SARS-CoV-2 IgM ELISA }\end{array}$ & $\lg M$ & Hospitalised & $\begin{array}{c}92.8(88.3-95 \cdot 7)^{b, c} \\
n=195 \\
C N(2), N L\end{array}$ & $\begin{array}{l}98.7(98.0-99.1) \\
\quad n=1,505 \\
C N(2), D K, N L(2)\end{array}$ \\
\hline ELISA & $\begin{array}{c}\text { Beijing Wantai Biological Pharmacy Enterprise, } \\
\text { Wantai SARS-CoV-2 total Ab ELISA }\end{array}$ & Total Ab & Hospitalised & $\begin{array}{l}97.5(95.9-98.5)^{c} \\
n=603 \\
\text { CN(2), DE, DK, NL }\end{array}$ & $\begin{array}{c}99.5(99.2-99.7) \\
n=3,097 \\
\mathrm{CN}(2), \mathrm{DE}, \mathrm{DK}(2), \mathrm{FR}(2), \\
\mathrm{NL}(3)\end{array}$ \\
\hline ELISA & $\begin{array}{c}\text { Beijing Wantai Biological Pharmacy Enterprise, } \\
\text { Wantai SARS-CoV-2 total Ab ELISA }\end{array}$ & Total Ab & Unk & $\begin{array}{c}97.5(94.9-98.8) \\
n=279 \\
\text { AT, DK, FR }\end{array}$ & Same as above \\
\hline ELISA & Bio-Rad, Platelia SARS-CoV-2 Total Ab & Total Ab & $\mathrm{Na}$ & $\mathrm{Nd}$ & $\begin{array}{c}96.4(93.3-98.1) \\
n=250 \\
B E, F R, L U, N L\end{array}$ \\
\hline LFIA, POC & CTK Biotech, OnSite COVID-19 IgG/IgM Rapid Test & $\lg G$ & $\mathrm{Na}$ & $\mathrm{Nd}$ & $\begin{array}{c}98.6(95.2-99.6) \\
n=148 \\
\mathrm{AU}, \mathrm{NL}\end{array}$ \\
\hline CLIA & $\begin{array}{c}\text { DiaSorin, Liaison XL S1/S2 IgG chemiluminescence } \\
\text { immunoassay }\end{array}$ & $\lg G$ & Hospitalised & $\begin{array}{c}92.9(89.6-95.2)^{b, c} \\
n=324 \\
C A, D E, N L\end{array}$ & $\begin{array}{c}97.7(97.3-98.0)^{c} \\
n=5,994 \\
\text { AT, BE(2), CA, DE(3), DK, } \\
\text { FI, FR, NL(2), SE, UK, US(2) }\end{array}$ \\
\hline CLIA & $\begin{array}{c}\text { DiaSorin, Liaison } X L S_{1} / S_{2} \text { IgG chemiluminescence } \\
\text { immunoassay }\end{array}$ & $\lg G$ & Mild/asymptomatic & $\begin{array}{c}81.9(76.3-86.3)^{b} \\
\qquad \begin{array}{c}\mathrm{b} \\
\mathrm{NL}, \mathrm{UK}\end{array} \\
\end{array}$ & Same as above \\
\hline
\end{tabular}

Ab: antibody; AT: Austria; AU: Australia; BE: Belgium; BR: Brazil; CA: Canada; CH: Switzerland; CLIA: chemiluminescence assay; CN: China; COVID-19: coronavirus disease; DE: Germany; DK: Denmark; ELISA: enzyme-linked immunosorbent assay; ES: Spain; FI: Finland; FR: France; GR: Greece; IT: Italy; JP: Japan; LFIA: lateral flow immunoassay; LU: Luxembourg; Na: not applicable; Nd: not determined, either due to no data or due to data from only one country or study; NL: The Netherlands; POC: point-of-care test; SARS-CoV-2: severe acute respiratory syndrome coronavirus 2; SE: Sweden; SG: Singapore; TW: Taiwan; UK: United Kingdom; Unk: unknown or unclearly defined; US: United States.

a Sensitivity and specificity values given as value (confidence interval), number of samples $(n=X)$, list of countries (number of studies per country if $>1)$. Value in bold if both confidence interval width $\leq 5 \%$ and value $\geq 95 \%$ (for sensitivity) or $\geq 98 \%$ (for specificity).

${ }^{\mathrm{b}}$ Confidence interval width $>5 \%$.

c Moderate study heterogeneity $(50.0 \leq 12<75.0 \%)$.

${ }^{d}$ High study heterogeneity $(\mid 2 \geq 75.0 \%)$

Only samples taken $>14$ days post onset of symptoms are included, and $\leq 28$ days post onset for IgM only as target. Rows are sorted alphabetically by test, target and case population. 
TABLE 2B

Pooled sensitivity and specificity results for SARS-CoV-2 antibody tests with confidence interval width $\leq 5 \%$ for either or both and based on at least two studies, up to 22 August 2020

\begin{tabular}{|c|c|c|c|c|c|}
\hline Category & Test & Target & Case population & Sensitivity ${ }^{a}$ & Specificity ${ }^{a}$ \\
\hline CLIA & $\begin{array}{c}\text { DiaSorin, Liaison XL S1/S2 IgG chemiluminescence } \\
\text { immunoassay }\end{array}$ & $\lg G$ & Unk & $\begin{array}{c}90.9(88.9-92.6)^{d} \\
n=967 \\
\text { AT(2), BE(2), DK, SE, } \\
\text { UK, US }\end{array}$ & Same as above \\
\hline CLIA & $\begin{array}{l}\text { Diazyme Laboratories, DZ-Lite SARS-CoV-2 IgM and } \\
\text { IgG CLIA }\end{array}$ & $\lg G$ & Unk & $\begin{array}{c}95.3(84.5-98.7)^{b} \\
n=43 \\
\text { US(2) }\end{array}$ & $\begin{array}{c}99.0(97.5-99.6) \\
n=414 \\
\text { US(2) }\end{array}$ \\
\hline CLIA & $\begin{array}{l}\text { Diazyme Laboratories, DZ-Lite SARS-CoV-2 IgM and } \\
\text { IgG CLIA }\end{array}$ & IgG or Ig $M$ & Unk & $\begin{array}{c}100.0(91.8-100.0)^{b} \\
n=43 \\
\text { US(2) }\end{array}$ & $\begin{array}{c}98.6(96.9-99.3) \\
n=414 \\
\text { US(2) }\end{array}$ \\
\hline CLIA & $\begin{array}{l}\text { Diazyme Laboratories, DZ-Lite SARS-CoV-2 IgM and } \\
\text { IgG CLIA }\end{array}$ & $\operatorname{Ig} M$ & Unk & $\begin{array}{c}90.7(78.4-96.3)^{b} \\
n=43 \\
\text { US(2) }\end{array}$ & $\begin{array}{c}99.5(98.3-99.9) \\
n=414 \\
\text { US(2) }\end{array}$ \\
\hline LFIA, POC & $\begin{array}{c}\text { Dynamiker Biotechnology Tianjin, } 2019 \text { nCoV IgG/ } \\
\text { IgM Rapid test }\end{array}$ & $\lg G$ or $\lg M$ & Hospitalised & $\begin{array}{c}100.0(89.0-100.0)^{b} \\
n=31 \\
B E, D K\end{array}$ & $\begin{array}{c}97.6(94.8-98.9) \\
n=248 \\
B E, D K, S E\end{array}$ \\
\hline LFIA, POC & $\begin{array}{c}\text { Dynamiker Biotechnology Tianjin, } 2019 \text { nCoV IgG/ } \\
\text { IgM Rapid test }\end{array}$ & IgG or Ig $M$ & Unk & $\begin{array}{c}89.0(79.8-94.3)^{b, d} \\
n=73 \\
S E, T W\end{array}$ & Same as above \\
\hline ELISA & $\begin{array}{l}\text { Epitope Diagnostics, EPI-KT-1032 Coronavirus } \\
\text { COVID-19 IgG ELISA Kit }\end{array}$ & $\lg G$ & Hospitalised & $\begin{array}{c}94.0(86.7-97.4)^{b, c} \\
n=83 \\
C A, N L, \text { US }\end{array}$ & $\begin{array}{c}97.6(96.7-98.3)^{c} \\
n=1,451 \\
\text { AT, CA, DE(2), NL, UK, } \\
\text { US(3) }\end{array}$ \\
\hline ELISA & $\begin{array}{l}\text { Epitope Diagnostics, EPI-KT-1032 Coronavirus } \\
\text { COVID-19 IgG ELISA Kit }\end{array}$ & $\lg G$ & Mild/asymptomatic & $\begin{array}{c}74.8(65.8-82.0)^{\mathrm{b}, \mathrm{d}} \\
\mathrm{n}=107 \\
\mathrm{NL}, \text { US }\end{array}$ & Same as above \\
\hline ELISA & $\begin{array}{l}\text { Epitope Diagnostics, EPI-KT-1032 Coronavirus } \\
\text { COVID-19 IgG ELISA Kit }\end{array}$ & $\operatorname{IgG}$ & Unk & $\begin{array}{c}96.0(90.1-98.4)^{b, c} \\
n=99 \\
\text { AT, DE, US }\end{array}$ & Same as above \\
\hline ELISA & $\begin{array}{l}\text { Epitope Diagnostics, EPI-KT-1033 Coronavirus } \\
\text { COVID-19 IgM ELISA Kit }\end{array}$ & $\lg M$ & Hospitalised & $\begin{array}{c}95.5(78.2-99.2)^{b, c} \\
n=22 \\
C A, N L\end{array}$ & $\begin{array}{l}98.1(97.0-98.9) \\
n=810 \\
\text { AT, CA, NL, US }\end{array}$ \\
\hline ELISA & $\begin{array}{l}\text { Epitope Diagnostics, EPI-KT-1033 Coronavirus } \\
\text { COVID-19 IgM ELISA Kit }\end{array}$ & $\lg M$ & Unk & $\begin{array}{c}83.3(70.4-91.3)^{b, c} \\
n=48 \\
\text { AT, US }\end{array}$ & Same as above \\
\hline
\end{tabular}

Ab: antibody; AT: Austria; AU: Australia; BE: Belgium; BR: Brazil; CA: Canada; CH: Switzerland; CLIA: chemiluminescence assay; CN: China; COVID-19: coronavirus disease; DE: Germany; DK: Denmark; ELISA: enzyme-linked immunosorbent assay; ES: Spain; FI: Finland; FR: France; GR: Greece; IT: Italy; JP: Japan; LFIA: lateral flow immunoassay; LU: Luxembourg; Na: not applicable; Nd: not determined, either due to no data or due to data from only one country or study; NL: The Netherlands; POC: point-of-care test; SARS-CoV-2: severe acute respiratory syndrome coronavirus 2; SE: Sweden; SG: Singapore; TW: Taiwan; UK: United Kingdom; Unk: unknown or unclearly defined; US: United States.

a Sensitivity and specificity values given as value (confidence interval), number of samples $(n=X)$, list of countries (number of studies per country if $>1)$. Value in bold if both confidence interval width $\leq 5 \%$ and value $\geq 95 \%$ (for sensitivity) or $\geq 98 \%$ (for specificity).

${ }^{\mathrm{b}}$ Confidence interval width $>5 \%$.

'Moderate study heterogeneity $(50.0 \leq 12<75.0 \%)$

${ }^{d}$ High study heterogeneity $(12 \geq 75.0 \%)$.

Only samples taken $>14$ days post onset of symptoms are included, and $\leq 28$ days post onset for IgM only as target. Rows are sorted alphabetically by test, target and case population. 
TABLE 2C

Pooled sensitivity and specificity results for SARS-CoV-2 antibody tests with confidence interval width $\leq 5 \%$ for either or both and based on at least two studies, up to 22 August 2020

\begin{tabular}{|c|c|c|c|c|c|}
\hline Category & Test & Target & Case population & Sensitivity $^{\mathrm{a}}$ & Specificity $^{a}$ \\
\hline ELISA & $\begin{array}{l}\text { Euroimmun Medizinische Labordiagnostika, Anti- } \\
\text { SARS-CoV-2 IgA S1 ELISA }\end{array}$ & $\operatorname{Ig} A$ & Hospitalised & $\begin{array}{c}96.0(92.5-97.9)^{b} \\
n=224 \\
B E(2), C A, D K, F I, F R, \\
\text { GR, NL }\end{array}$ & $\begin{array}{c}86.7(84.9-88.3)^{d} \\
n=1,459 \\
A U, B E(2), C A, D K, E S, \\
F I(2), F R(2), G R, L U, N L(2), \\
\text { US }\end{array}$ \\
\hline ELISA & $\begin{array}{l}\text { Euroimmun Medizinische Labordiagnostika, Anti- } \\
\text { SARS-CoV-2 IgA S1 ELISA }\end{array}$ & $\lg A$ & Mild/asymptomatic & $\begin{array}{c}67.2(55.0-77.4)^{b} \\
n=64 \\
\mathrm{FI}, \mathrm{NL}\end{array}$ & Same as above \\
\hline ELISA & $\begin{array}{l}\text { Euroimmun Medizinische Labordiagnostika, Anti- } \\
\text { SARS-CoV-2 IgA S1 ELISA }\end{array}$ & $\lg A$ & Unk & $\begin{array}{c}94.8(90.9-97.1)^{b} \\
n=212 \\
A U, B E, F R, U S\end{array}$ & Same as above \\
\hline ELISA & $\begin{array}{c}\text { Euroimmun Medizinische Labordiagnostika, Anti- } \\
\text { SARS-CoV-2 IgG S1 ELISA }\end{array}$ & $\lg G$ & Hospitalised & $\begin{array}{r}92.6(89.7-94.7) \\
n=431 \\
B E(3), C A, C H(2), D E, \\
D K, F I, F R, G R, N L, U S\end{array}$ & $\begin{array}{c}97.9(97.4-98.3) \\
n=3,954 \\
A U, B E(3), C A, C H(2), \\
D E(6), D K(2), E S, F I(2), \\
F R(3), G R, L U, N L(2), U S(5)\end{array}$ \\
\hline ELISA & $\begin{array}{c}\text { Euroimmun Medizinische Labordiagnostika, Anti- } \\
\text { SARS-CoV-2 IgG S1 ELISA }\end{array}$ & $\lg G$ & Mild/asymptomatic & $\begin{array}{c}79.5(71.9-85.5)^{b, d} \\
n=132 \\
C H, F I, N L, \text { US }\end{array}$ & Same as above \\
\hline ELISA & $\begin{array}{l}\text { Euroimmun Medizinische Labordiagnostika, Anti- } \\
\text { SARS-CoV-2 IgG S1 ELISA }\end{array}$ & $\lg G$ & Unk & $\begin{array}{c}89.0(86.7-91.0)^{c} \\
n=785 \\
\text { AT, AU, BE, DE(2), DK, } \\
\text { FR, UK, US(2) }\end{array}$ & Same as above \\
\hline LFIA, POC & $\begin{array}{l}\text { Getein Biotech, One Step Test for Novel Coronavirus } \\
(2019-\mathrm{nCoV}) \text { IgM/IgG Antibody (Colloidal Gold) }\end{array}$ & $\lg G$ & $\mathrm{Na}$ & $\mathrm{Nd}$ & $\begin{array}{c}100.0(96.9-100.0) \\
n=120 \\
\text { CA, US }\end{array}$ \\
\hline LFIA, POC & $\begin{array}{l}\text { Getein Biotech, One Step Test for Novel Coronavirus } \\
\text { (2019-nCoV) IgM/IgG Antibody (Colloidal Gold) }\end{array}$ & $\lg G$ or $\lg M$ & $\mathrm{Na}$ & $\mathrm{Nd}$ & $\begin{array}{c}99.2(95.4-99.9) \\
n=120 \\
\text { CA, US }\end{array}$ \\
\hline LFIA, POC & $\begin{array}{l}\text { Getein Biotech, One Step Test for Novel Coronavirus } \\
(2019-\mathrm{nCoV}) \text { IgM/IgG Antibody (Colloidal Gold) }\end{array}$ & $\lg M$ & $\mathrm{Na}$ & $\mathrm{Nd}$ & $\begin{array}{c}99.2(95.4-99.9) \\
n=120 \\
\text { CA, US }\end{array}$ \\
\hline LFIA, POC & $\begin{array}{c}\text { Guangzhou Wondfo Biotech, Wondfo SARS-CoV-2 } \\
\text { Antibody Test }\end{array}$ & $\lg G$ or Ig $M$ & Unk & $\begin{array}{c}88.0(82.6-92.0)^{b, d} \\
n=184 \\
\text { AU, ES, TW, US }\end{array}$ & $\begin{array}{c}99.3(98.3-99.7) \\
n=605 \\
A U, B R, E S, U S(2)\end{array}$ \\
\hline LFIA, POC & $\begin{array}{c}\text { Hangzhou Alltest Biotech, 2019-nCoV IgG/IgM Rapid } \\
\text { Test Cassette }\end{array}$ & $\lg G$ & Unk & $\begin{array}{c}88.7(81.6-93.3)^{b} \\
n=115 \\
A U, E S\end{array}$ & $\begin{array}{c}100.0(98.5-100.0) \\
n=254 \\
A U, E S(2)\end{array}$ \\
\hline
\end{tabular}

Ab: antibody; AT: Austria; AU: Australia; BE: Belgium; BR: Brazil; CA: Canada; CH: Switzerland; CLIA: chemiluminescence assay; CN: China; COVID-19: coronavirus disease; DE: Germany; DK: Denmark; ELISA: enzyme-linked immunosorbent assay; ES: Spain; FI: Finland; FR: France; GR: Greece; IT: Italy; JP: Japan; LFIA: lateral flow immunoassay; LU: Luxembourg; Na: not applicable; Nd: not determined, either due to no data or due to data from only one country or study; NL: The Netherlands; POC: point-of-care test; SARS-CoV-2: severe acute respiratory syndrome coronavirus 2; SE: Sweden; SG: Singapore; TW: Taiwan; UK: United Kingdom; Unk: unknown or unclearly defined; US: United States.

a Sensitivity and specificity values given as value (confidence interval), number of samples $(n=X)$, list of countries (number of studies per country if $>1)$. Value in bold if both confidence interval width $\leq 5 \%$ and value $\geq 95 \%$ (for sensitivity) or $\geq 98 \%$ (for specificity).

${ }^{\mathrm{b}}$ Confidence interval width $>5 \%$.

c Moderate study heterogeneity $(50.0 \leq 12<75.0 \%)$.

${ }^{d}$ High study heterogeneity $(12 \geq 75.0 \%)$.

Only samples taken $>14$ days post onset of symptoms are included, and $\leq 28$ days post onset for IgM only as target. Rows are sorted alphabetically by test, target and case population. 
TABLE 2D

Pooled sensitivity and specificity results for SARS-CoV-2 antibody tests with confidence interval width $\leq 5 \%$ for either or both and based on at least two studies, up to 22 August 2020

\begin{tabular}{|c|c|c|c|c|c|}
\hline Category & Test & Target & Case population & Sensitivity $^{\mathrm{a}}$ & Specificity $^{a}$ \\
\hline LFIA, POC & $\begin{array}{c}\text { Hangzhou Alltest Biotech, 2019-nCoV IgG/IgM Rapid } \\
\text { Test Cassette }\end{array}$ & IgG or Ig $M$ & Unk & $\begin{array}{c}92.3(87.2-95.4)^{b} \\
n=168 \\
\mathrm{AU}, \mathrm{ES}, \mathrm{TW}\end{array}$ & $\begin{array}{c}96.7(93.8-98.2) \\
n=269 \\
A U, D K, E S(2)\end{array}$ \\
\hline LFIA, POC & $\begin{array}{c}\text { Hangzhou Alltest Biotech, 2019-nCoV IgG/IgM Rapid } \\
\text { Test Cassette }\end{array}$ & $\lg M$ & Unk & $\begin{array}{c}21.7(15.2-30.1)^{b, d} \\
n=115 \\
A U, E S\end{array}$ & $\begin{array}{c}97.2(94.4-98.7) \\
n=254 \\
A U, E S(2)\end{array}$ \\
\hline LFIA, POC & $\begin{array}{l}\text { Innovita Biological Technology, 2019-nCoV Ab Test } \\
\text { (Colloidal Gold) }\end{array}$ & $\lg G$ & Hospitalised & $\begin{array}{c}86.9(76.2-93.2)^{b} \\
n=61 \\
\text { CA, JP }\end{array}$ & $\begin{array}{c}100.0(98.5-100.0) \\
n=258 \\
\text { CA, JP, US }\end{array}$ \\
\hline LFIA, POC & $\begin{array}{l}\text { Innovita Biological Technology, 2019-nCoV Ab Test } \\
\text { (Colloidal Gold) }\end{array}$ & $\operatorname{Ig} M$ & Hospitalised & $\begin{array}{c}75.4(63.3-84 \cdot 5)^{b, d} \\
n=61 \\
\text { CA, JP }\end{array}$ & $\begin{array}{c}98.4(96.1-99.4) \\
n=258 \\
\text { CA, JP, US }\end{array}$ \\
\hline ELISA & Mikrogen Diagnostik, recomWell SARS-CoV-2 IgG & $\lg G$ & $\mathrm{Na}$ & $\mathrm{Nd}$ & $\begin{array}{c}96.4(94.2-97.8) \\
n=445 \\
B E, D E, N L\end{array}$ \\
\hline ELISA & $\begin{array}{l}\text { NovaTec Immundiagnostica, NovaLisa SARS-CoV-2 } \\
\text { IgA ELISA }\end{array}$ & $\lg A$ & Hospitalised & $\begin{array}{c}88.7(78.5-94.4)^{b} \\
n=62 \\
B E(2)\end{array}$ & $\begin{array}{c}95.2(92.1-97.1)^{c} \\
n=293 \\
B E(2), I T, N L\end{array}$ \\
\hline ELISA & $\begin{array}{c}\text { NovaTec Immundiagnostica, NovaLisa SARS-CoV-2 } \\
\text { IgG ELISA }\end{array}$ & $\lg G$ & Hospitalised & $\begin{array}{c}91.9(82.5-96.5)^{b} \\
n=62 \\
B E(2)\end{array}$ & $\begin{array}{c}97.3(94.7-98.6) \\
n=293 \\
B E(2), I T, N L\end{array}$ \\
\hline ELISA & $\begin{array}{c}\text { NovaTec Immundiagnostica, NovaLisa SARS-CoV-2 } \\
\text { IgM ELISA }\end{array}$ & $\lg M$ & Hospitalised & $\begin{array}{c}43.5(31.9-55.9)^{b, d} \\
n=62 \\
B E(2)\end{array}$ & $\begin{array}{c}99.0(97.0-99.7) \\
n=293 \\
B E(2), I T, N L\end{array}$ \\
\hline CLIA & $\begin{array}{c}\text { Ortho Clinical Diagnostics, VITROS } \\
\text { Immunodiagnostic Products Anti-SARS-CoV-2 IgG }\end{array}$ & $\lg G$ & Unk & $\begin{array}{c}93.4(89.4-96.0)^{b} \\
n=227 \\
\text { DK, UK }\end{array}$ & $\begin{array}{c}99.7(99.3-99.9) \\
n=1,420 \\
\text { DK, UK, US }\end{array}$ \\
\hline CLIA & $\begin{array}{c}\text { Ortho Clinical Diagnostics, VITROS } \\
\text { Immunodiagnostic Products Anti-SARS-CoV-2 Total } \\
\text { Ab }\end{array}$ & Total Ab & $\mathrm{Na}$ & $\mathrm{Nd}$ & $\begin{array}{c}100.0\left(99.5^{-100.0)}\right. \\
n=732 \\
\text { DK, US }\end{array}$ \\
\hline CLIA & Roche, Elecsys Anti-SARS-CoV-2 & Total Ab & Hospitalised & $\begin{array}{c}85.7(75.7-92.1)^{b} \\
n=70 \\
C A, D E, N L\end{array}$ & $\begin{array}{c}99.8(99.7-99.9) \\
n=7,833 \\
\text { AT, BE(3), CA, DE(5), DK, } \\
\text { LU, NL, SE, SG, UK(2), } \\
\text { US(5) }\end{array}$ \\
\hline
\end{tabular}

Ab: antibody; AT: Austria; AU: Australia; BE: Belgium; BR: Brazil; CA: Canada; CH: Switzerland; CLIA: chemiluminescence assay; CN: China; COVID-19: coronavirus disease; DE: Germany; DK: Denmark; ELISA: enzyme-linked immunosorbent assay; ES: Spain; FI: Finland; FR: France; GR: Greece; IT: Italy; JP: Japan; LFIA: lateral flow immunoassay; LU: Luxembourg; Na: not applicable; Nd: not determined, either due to no data or due to data from only one country or study; NL: The Netherlands; POC: point-of-care test; SARS-CoV-2: severe acute respiratory syndrome coronavirus 2; SE: Sweden; SG: Singapore; TW: Taiwan; UK: United Kingdom; Unk: unknown or unclearly defined; US: United States.

a Sensitivity and specificity values given as value (confidence interval), number of samples $(n=X)$, list of countries (number of studies per country if $>1)$. Value in bold if both confidence interval width $\leq 5 \%$ and value $\geq 95 \%$ (for sensitivity) or $\geq 98 \%$ (for specificity).

${ }^{\mathrm{b}}$ Confidence interval width $>5 \%$.

${ }^{c}$ Moderate study heterogeneity $(50.0 \leq 12<75.0 \%)$.

${ }^{d}$ High study heterogeneity ( $\left.\mid 2 \geq 75.0 \%\right)$.

Only samples taken $>14$ days post onset of symptoms are included, and $\leq 28$ days post onset for IgM only as target. Rows are sorted alphabetically by test, target and case population. 
TABLE 2E

Pooled sensitivity and specificity results for SARS-CoV-2 antibody tests with confidence interval width $\leq 5 \%$ for either or both and based on at least two studies, up to 22 August 2020

\begin{tabular}{|c|c|c|c|c|c|}
\hline Category & Test & Target & Case population & Sensitivity $^{\mathrm{a}}$ & Specificity $^{a}$ \\
\hline CLIA & Roche, Elecsys Anti-SARS-CoV-2 & Total Ab & Mild/asymptomatic & $\begin{array}{c}91.7(84.4-95 \cdot 7)^{b, c} \\
n=96 \\
N L, \text { UK }\end{array}$ & Same as above \\
\hline CLIA & Roche, Elecsys Anti-SARS-CoV-2 & Total Ab & Unk & $\begin{array}{c}94.7(93.3-95.7)^{c} \\
n=1,351 \\
\text { AT(2), BE(3), DE(2), DK, } \\
\text { SE, SG, UK(2), US(2) }\end{array}$ & Same as above \\
\hline LFIA, POC & SD BioSensor, Standard Q COVID-19 IgM/IgG Duo & $\lg G$ & $\mathrm{Na}$ & $\mathrm{Nd}$ & $\begin{array}{c}99.8(99.3-99.9)^{c} \\
n=1,254 \\
\text { US(2) }\end{array}$ \\
\hline LFIA, POC & SD BioSensor, Standard Q COVID-19 IgM/IgG Duo & $\lg M$ & $\mathrm{Na}$ & $\mathrm{Nd}$ & $\begin{array}{c}98.8(98.0-99.3) \\
n=1,256 \\
\text { US }(2)\end{array}$ \\
\hline CLIA & $\begin{array}{c}\text { Shenzhen New Industries Biomedical Engineering } \\
\text { (SNIBE), Maglumi 2019-nCoV (SARS-CoV-2) IgG/ } \\
\text { IgM kit }\end{array}$ & $\lg G$ & Hospitalised & $\begin{array}{c}93.4(85.5-97.2)^{\mathrm{b}, \mathrm{c}} \\
\mathrm{n}=76 \\
\mathrm{BE}(2)\end{array}$ & $\begin{array}{c}97.6(96.8-98.3)^{d} \\
n=1,744 \\
B E(2), C N(2), D K\end{array}$ \\
\hline CLIA & $\begin{array}{c}\text { Shenzhen New Industries Biomedical Engineering } \\
\text { (SNIBE), Maglumi 2019-nCoV (SARS-CoV-2) IgG/ } \\
\text { IgM kit }\end{array}$ & $\lg G$ & Unk & $\begin{array}{c}91.1(89.2-92.6)^{d} \\
n=1084 \\
\text { CN, DK }\end{array}$ & Same as above \\
\hline CLIA & $\begin{array}{c}\text { Shenzhen New Industries Biomedical Engineering } \\
\text { (SNIBE), Maglumi 2019-nCoV (SARS-CoV-2) IgG/ } \\
\text { IgM kit }\end{array}$ & $\operatorname{Ig} G$ or $\lg M$ & Hospitalised & $\begin{array}{c}96.1(89.0-98.6)^{b} \\
n=76 \\
B E(2)\end{array}$ & $\begin{array}{c}98.6(96.4-99.5) \\
n=285 \\
B E(3)\end{array}$ \\
\hline CLIA & $\begin{array}{c}\text { Shenzhen New Industries Biomedical Engineering } \\
\text { (SNIBE), Maglumi 2019-nCoV (SARS-CoV-2) IgG/ } \\
\text { IgM kit }\end{array}$ & $\lg M$ & Hospitalised & $\begin{array}{c}93.4(85.5-97.2)^{b, c} \\
n=76 \\
B E(2)\end{array}$ & $\begin{array}{c}99.2(98.7-99.5)^{d} \\
n=1,756 \\
B E(2), C N(2), D K\end{array}$ \\
\hline CLIA & $\begin{array}{c}\text { Shenzhen New Industries Biomedical Engineering } \\
\text { (SNIBE), Maglumi 2019-nCoV (SARS-CoV-2) IgG/ } \\
\text { IgM kit }\end{array}$ & $\lg M$ & Unk & $\begin{array}{c}67.8(65.0-70.5)^{b, d} \\
n=1084 \\
\text { CN, DK }\end{array}$ & Same as above \\
\hline CLIA & $\begin{array}{c}\text { Shenzhen Yahuilong (YHLO) Biotech, SARS-CoV-2 } \\
\text { IgG/IgM antibody detection kit }\end{array}$ & $\lg G$ & $\mathrm{Na}$ & $\mathrm{Nd}$ & $\begin{array}{c}99.0(98.3-99.4) \\
n=1,313 \\
C N(2), D K, \text { IT }\end{array}$ \\
\hline CLIA & $\begin{array}{c}\text { Shenzhen Yahuilong (YHLO) Biotech, SARS-CoV-2 } \\
\text { IgG/IgM antibody detection kit }\end{array}$ & $\lg M$ & $\mathrm{Na}$ & $\mathrm{Nd}$ & $\begin{array}{c}98.7(97.9-99.2)^{d} \\
n=1314 \\
\text { CN(2), DK, IT }\end{array}$ \\
\hline
\end{tabular}

Ab: antibody; AT: Austria; AU: Australia; BE: Belgium; BR: Brazil; CA: Canada; CH: Switzerland; CLIA: chemiluminescence assay; CN: China; COVID-19: coronavirus disease; DE: Germany; DK: Denmark; ELISA: enzyme-linked immunosorbent assay; ES: Spain; FI: Finland; FR: France; GR: Greece; IT: Italy; JP: Japan; LFIA: lateral flow immunoassay; LU: Luxembourg; Na: not applicable; Nd: not determined, either due to no data or due to data from only one country or study; NL: The Netherlands; POC: point-of-care test; SARS-CoV-2: severe acute respiratory syndrome coronavirus 2; SE: Sweden; SG: Singapore; TW: Taiwan; UK: United Kingdom; Unk: unknown or unclearly defined; US: United States.

a Sensitivity and specificity values given as value (confidence interval), number of samples $(n=X)$, list of countries (number of studies per country if $>1)$. Value in bold if both confidence interval width $\leq 5 \%$ and value $\geq 95 \%$ (for sensitivity) or $\geq 98 \%$ (for specificity).

${ }^{\mathrm{b}}$ Confidence interval width $>5 \%$.

${ }^{c}$ Moderate study heterogeneity $(50.0 \leq 12<75.0 \%)$.

${ }^{d}$ High study heterogeneity $(\mid 2 \geq 75.0 \%)$.

Only samples taken $>14$ days post onset of symptoms are included, and $\leq 28$ days post onset for IgM only as target. Rows are sorted alphabetically by test, target and case population. 
TABLE 2F

Pooled sensitivity and specificity results for SARS-CoV-2 antibody tests with confidence interval width $\leq 5 \%$ for either or both and based on at least two studies, up to 22 August 2020

\begin{tabular}{|c|c|c|c|c|c|}
\hline Category & Test & Target & Case population & Sensitivity $^{\mathrm{a}}$ & Specificity $^{a}$ \\
\hline CLIA & $\begin{array}{c}\text { Siemens, Healthineers SARS-CoV-2 Total Assay on } \\
\text { Atellica/ADVIA Centaur }\end{array}$ & Total Ab & Unk & $\begin{array}{c}96.7(95.2-97.8)^{d} \\
n=757 \\
\text { DE, DK, UK }\end{array}$ & $\begin{array}{c}99.8(99.5-99.9) \\
n=2,108 \\
D E(2), D K, U K\end{array}$ \\
\hline LFIA, POC & $\begin{array}{c}\text { SureScreen Diagnostic, Covid-19 IgG/IgM Rapid Test } \\
\text { Cassette }\end{array}$ & $\lg G$ & $\mathrm{Na}$ & $\mathrm{Nd}$ & $\begin{array}{c}99.0(96.4-99.7) \\
n=198 \\
B E, N L\end{array}$ \\
\hline LFIA, POC & $\begin{array}{c}\text { VivaChek Biotech, VivaDiag COVID-19 IgM/IgG } \\
\text { Rapid Test }\end{array}$ & $\lg G$ & Unk & $\begin{array}{c}78.9(69.7-85.9)^{b} \\
n=95 \\
A U, \text { US }\end{array}$ & $\begin{array}{c}98.2(96.1-99.2) \\
n=334 \\
A U, B E, I T, N L, U S\end{array}$ \\
\hline LFIA, POC & $\begin{array}{c}\text { VivaChek Biotech, VivaDiag COVID-19 IgM/IgG } \\
\text { Rapid Test }\end{array}$ & IgG or Ig $M$ & Hospitalised & $\begin{array}{c}100.0(89.0-100.0)^{b} \\
n=31 \\
B E, N L\end{array}$ & $\begin{array}{c}97.5(95.2-98.7) \\
n=324 \\
A U, B E, I T, \text { US }\end{array}$ \\
\hline LFIA, POC & $\begin{array}{c}\text { VivaChek Biotech, VivaDiag COVID-19 IgM/IgG } \\
\text { Rapid Test }\end{array}$ & IgG or IgM & Unk & $\begin{array}{c}80.0(70.9-86.8)^{b} \\
n=95 \\
\text { AU, US }\end{array}$ & Same as above \\
\hline LFIA, POC & $\begin{array}{c}\text { VivaChek Biotech, VivaDiag COVID-19 IgM/IgG } \\
\text { Rapid Test }\end{array}$ & $\operatorname{Ig} M$ & Unk & $\begin{array}{c}80.0(70.9-86.8)^{b} \\
n=95 \\
A U, \text { US }\end{array}$ & $\begin{array}{c}97.8(95.6-98.9) \\
n=324 \\
A U, B E, I T, U S\end{array}$ \\
\hline LFIA, POC & $\begin{array}{c}\text { Xiamen Biotime Biotechnology, SARS-CoV-2 IgG/ } \\
\text { IgM Rapid Qualitative Test Kit }\end{array}$ & $\lg G$ & $\mathrm{Na}$ & $\mathrm{Nd}$ & $\begin{array}{c}98.0(94 \cdot 3-99 \cdot 3) \\
n=150 \\
\text { FI, US }\end{array}$ \\
\hline CLIA & $\begin{array}{l}\text { Xiamen Innodx Biotech, Antibody test kit for } \\
\text { 2019-nCoV }\end{array}$ & $\lg G$ or $\lg M$ & $\mathrm{Na}$ & $\mathrm{Nd}$ & $\begin{array}{c}99.3(98.0-99.8) \\
n=430 \\
\mathrm{CN}(2)\end{array}$ \\
\hline LFIA, POC & $\begin{array}{c}\text { Zhejiang Orient Gene Biotech, COVID-19 IgG/IgM } \\
\text { Rapid Test Cassette }\end{array}$ & $\lg G$ & Hospitalised & $\begin{array}{c}96.7(91.7-98.7)^{b} \\
n=120 \\
B E, C H, N L\end{array}$ & $\begin{array}{c}97.7(96.1-98.7) \\
n=568 \\
\text { BE, CH, FR, NL, SE }\end{array}$ \\
\hline LFIA, POC & $\begin{array}{c}\text { Zhejiang Orient Gene Biotech, COVID-19 IgG/IgM } \\
\text { Rapid Test Cassette }\end{array}$ & $\lg G$ & Unk & $\begin{array}{c}92.4(85.1-96.3)^{b} \\
n=92 \\
F R, S E\end{array}$ & Same as above \\
\hline LFIA, POC & $\begin{array}{c}\text { Zhejiang Orient Gene Biotech, COVID-19 IgG/IgM } \\
\text { Rapid Test Cassette }\end{array}$ & $\operatorname{Ig} M$ & Hospitalised & $\begin{array}{c}86.0(77.5-91.6)^{b} \\
n=93 \\
B E, N L\end{array}$ & $\begin{array}{c}98.4(96.3-99.3) \\
n=308 \\
B E, F R, S E\end{array}$ \\
\hline
\end{tabular}

Ab: antibody; AT: Austria; AU: Australia; BE: Belgium; BR: Brazil; CA: Canada; CH: Switzerland; CLIA: chemiluminescence assay; CN: China; COVID-19: coronavirus disease; DE: Germany; DK: Denmark; ELISA: enzyme-linked immunosorbent assay; ES: Spain; FI: Finland; FR: France; GR: Greece; IT: Italy; JP: Japan; LFIA: lateral flow immunoassay; LU: Luxembourg; Na: not applicable; Nd: not determined, either due to no data or due to data from only one country or study; NL: The Netherlands; POC: point-of-care test; SARS-CoV-2: severe acute respiratory syndrome coronavirus 2; SE: Sweden; SG: Singapore; TW: Taiwan; UK: United Kingdom; Unk: unknown or unclearly defined; US: United States.

a Sensitivity and specificity values given as value (confidence interval), number of samples $(n=X)$, list of countries (number of studies per country if $>1)$. Value in bold if both confidence interval width $\leq 5 \%$ and value $\geq 95 \%$ (for sensitivity) or $\geq 98 \%$ (for specificity).

${ }^{\mathrm{b}}$ Confidence interval width $>5 \%$.

c Moderate study heterogeneity $(50.0 \leq 12<75.0 \%)$

${ }^{d}$ High study heterogeneity $(12 \geq 75.0 \%)$.

Only samples taken $>14$ days post onset of symptoms are included, and $\leq 28$ days post onset for IgM only as target. Rows are sorted alphabetically by test, target and case population. 
TABLE 2G

Pooled sensitivity and specificity results for SARS-CoV-2 antibody tests with confidence interval width $\leq 5 \%$ for either or both and based on at least two studies, up to 22 August 2020

\begin{tabular}{|c|c|c|c|c|c|}
\hline Category & Test & Target & Case population & Sensitivity $^{\mathrm{a}}$ & Specificity $^{a}$ \\
\hline LFIA, POC & $\begin{array}{c}\text { Zhejiang Orient Gene Biotech, COVID-19 IgG/IgM } \\
\text { Rapid Test Cassette }\end{array}$ & $\lg M$ & Unk & $\begin{array}{c}82.6(73.6-89.0)^{b, c} \\
n=92 \\
F R, S E\end{array}$ & Same as above \\
\hline LFIA, POC & $\begin{array}{c}\text { Zhuhai Livzon Pharmaceutical Group, Diagnostic Kit } \\
\text { for IgM / IgG Antibody to Coronavirus (SARS-CoV-2) } \\
\text { (Lateral Flow) }\end{array}$ & IgG & Hospitalised & $\begin{array}{c}86.4(80.3-90.9)^{b} \\
n=162 \\
C N(2), F R\end{array}$ & $\begin{array}{c}98.0(94 \cdot 3-99 \cdot 3) \\
n=150 \\
\text { CN, FR, US }\end{array}$ \\
\hline LFIA, POC & $\begin{array}{c}\text { Zhuhai Livzon Pharmaceutical Group, Diagnostic Kit } \\
\text { for IgM / IgG Antibody to Coronavirus (SARS-CoV-2) } \\
\text { (Lateral Flow) }\end{array}$ & $\lg M$ & Hospitalised & $\begin{array}{c}75.9(68.8-81.9)^{b} \\
n=162 \\
\mathrm{CN}(2), \mathrm{FR}\end{array}$ & $\begin{array}{c}99.3(96.3-99.9) \\
n=150 \\
\text { CN, FR, US }\end{array}$ \\
\hline
\end{tabular}

Ab: antibody; AT: Austria; AU: Australia; BE: Belgium; BR: Brazil; CA: Canada; CH: Switzerland; CLIA: chemiluminescence assay; CN: China; COVID-19: coronavirus disease; DE: Germany; DK: Denmark; ELISA: enzyme-linked immunosorbent assay; ES: Spain; FI: Finland; FR: France; GR: Greece; IT: Italy; JP: Japan; LFIA: lateral flow immunoassay; LU: Luxembourg; Na: not applicable; Nd: not determined, either due to no data or due to data from only one country or study; NL: The Netherlands; POC: point-of-care test; SARS-CoV-2: severe acute respiratory syndrome coronavirus 2; SE: Sweden; SG: Singapore; TW: Taiwan; UK: United Kingdom; Unk: unknown or unclearly defined; US: United States.

a Sensitivity and specificity values given as value (confidence interval), number of samples $(n=X)$, list of countries (number of studies per country if $>1)$. Value in bold if both confidence interval width $\leq 5 \%$ and value $\geq 95 \%$ (for sensitivity) or $\geq 98 \%$ (for specificity).

${ }^{b}$ Confidence interval width $>5 \%$.

c Moderate study heterogeneity $(50.0 \leq 12<75.0 \%)$.

${ }^{d}$ High study heterogeneity $(12 \geq 75.0 \%)$.

Only samples taken $>14$ days post onset of symptoms are included, and $\leq 28$ days post onset for IgM only as target. Rows are sorted alphabetically by test, target and case population.

confidence interval ranges. It is reassuring that the clinical performance of several nucleic acid and antibody tests exceeded the minimum performance criteria. As time progresses, the list of tests with sufficient available performance data is expected to grow.

At the same time, the available evidence for point-ofcare nucleic acid and antigen tests remains scarce, even though these tests can have substantial practical advantages for e.g. screening. We therefore recommend more emphasis on the validation of these tests, including as part of a testing algorithm, whereby the sensitivity and specificity of taking two tests with a number of days in between is assessed, and which can for example be useful to reduce the duration of a quarantine period.

The comparison between the independently assessed clinical performance data and manufacturer-reported clinical performance revealed that in particular sensitivity is frequently ( $34.4 \%$ of the cases in this study) significantly overestimated by the manufacturer. At a minimum, this emphasises that such independent assessments are clearly necessary. In the longer term, an explicit and proactive regulatory mechanism in Europe to compare available independently generated evidence on these tests against the manufacturerreported values, coupled with appropriate regulatory action, would be useful. This could also be rewarding towards those manufacturers that do provide robust estimates of their product's performance. The new in vitro diagnostic medical devices Regulation (EU) 2017/746 (IVDR), which will enter into force in May 2022, will impose more stringent requirements on clinical performance studies done by manufacturers. In addition, the IVDR will also regulate the use of lab-developed tests such as the in-house PCR tests developed for COVID-19 [25]. Because of the COVID-19 pandemic, the European Commission has recently proposed to modify the roll-out [26].

Limitations of our article include that most of the included studies had a substantial risk of bias in the sample selection, especially for the sensitivity panel, as established also in the assessments performed in the systematic reviews that we used as a source. Results were mainly based on hospitalised cases or poorly defined populations, whereas the population of interest often consists of symptomatic cases in general, or even asymptomatic cases, and differences in performance may exist depending on disease severity. Performance also varies depending on the type of specimen used, and our study design allowed for the inclusion of multiple specimen types in accordance with the instructions for use. This reflected to some extent clinical practice, but is also a contributing factor to study heterogeneity that we did not address here. Similarly, the pre-analytical steps such as RNA extraction can 


\section{TABLE 3A}

Pooled positive agreement and specificity results for SARS-CoV-2 nucleic acid tests with confidence interval width $\leq 5 \%$ for either or both and based on at least two studies, up to 22 August 2020

\begin{tabular}{|c|c|c|c|c|c|}
\hline Category & Test & Target & $\begin{array}{l}\text { Case } \\
\text { population }\end{array}$ & Positive agreement ${ }^{a}$ & Specificity $^{\mathrm{a}}$ \\
\hline PCR & $\begin{array}{l}\text { Altona Diagnostics, RealStar SARS-CoV-2 RT-PCR } \\
\text { Kit } 1.0\end{array}$ & $\mathrm{E}$ & Unk & $\begin{array}{c}88.1(80.4-93.1)^{b} \\
n=101 \\
\text { CH, FR, NL, US }\end{array}$ & $\begin{array}{c}100.0(96.7-100.0) \\
n=112 \\
\mathrm{CH}, \mathrm{NL}\end{array}$ \\
\hline PCR & $\begin{array}{l}\text { Altona Diagnostics, RealStar SARS-CoV-2 RT-PCR } \\
\text { Kit } 1.0\end{array}$ & S & Unk & $\begin{array}{c}87.1(79.2-92.3)^{b} \\
n=101 \\
\text { CH, FR, NL, US }\end{array}$ & $\begin{array}{c}100.0(96.7-100.0) \\
n=112 \\
\mathrm{CH}, \mathrm{NL}\end{array}$ \\
\hline PCR & $\begin{array}{l}\text { Altona Diagnostics, RealStar SARS-CoV-2 RT-PCR } \\
\text { Kit } 1.0\end{array}$ & $\mathrm{~S}$ or $\mathrm{E}$ & Unk & $\begin{array}{c}81.6(75.8-86.3)^{b, c} \\
n=207 \\
F R(3), N L\end{array}$ & $\begin{array}{c}100.0(98.4-100.0) \\
n=237 \\
F R, N L, U K\end{array}$ \\
\hline PCR & AusDiagnostics, Coronavirus Typing Assay & ORF1ab & $\mathrm{Na}$ & $\mathrm{Nd}$ & $\begin{array}{c}100.0(98.5-100.0) \\
n=254 \\
A U, \text { UK }\end{array}$ \\
\hline PCR & $\begin{array}{l}\text { BGI, Real-time fluorescent RT-PCR kit for detecting } \\
\qquad 2019 \mathrm{nCoV}\end{array}$ & ORF1ab & Unk & $\begin{array}{c}93.8(88.7-96.7)^{b} \\
n=146 \\
\mathrm{CH}, \mathrm{JP}, \mathrm{NL}, \mathrm{PL}\end{array}$ & $\begin{array}{c}99.1(95.1-99.8) \\
n=112 \\
\mathrm{CH}, \mathrm{NL}\end{array}$ \\
\hline PCR, POC & Cepheid, GeneXpert Xpert Xpress SARS-CoV-2 & $\mathrm{E}$ or $\mathrm{N}$ & Unk & $\begin{array}{c}98.8(97.3-99.5) \\
n=427 \\
\text { BE, CH, CY, DE, FI, FR, NL, SE, } \\
\text { US(5) }\end{array}$ & $\begin{array}{c}100.0(82.4-100.0) b \\
n=18 \\
\text { BE, CH, SE }\end{array}$ \\
\hline PCR & $\begin{array}{c}\text { CerTest Biotec, VIASURE SARS-CoV-2 Real Time PCR } \\
\text { Detection Kit }\end{array}$ & $\mathrm{N}$ & Unk & $\begin{array}{c}96.8(89.1-99.1)^{\mathrm{b}, \mathrm{c}} \\
\mathrm{n}=63 \\
\mathrm{CH}, \mathrm{NL}\end{array}$ & $\begin{array}{c}100.0(96.7-100.0) \\
n=112 \\
\mathrm{CH}, \mathrm{NL}\end{array}$ \\
\hline PCR & $\begin{array}{c}\text { CerTest Biotec, VIASURE SARS-CoV-2 Real Time PCR } \\
\text { Detection Kit }\end{array}$ & ORF1ab or $\mathrm{N}$ & $\mathrm{Na}$ & $\mathrm{Nd}$ & $\begin{array}{c}100.0(98.2-100.0) \\
n=207 \\
\text { NL, UK }\end{array}$ \\
\hline PCR & DiaSorin, Simplexa COVID-19 Direct RT-PCR Kit & ORF1ab or S & Unk & $\begin{array}{c}97.8(94.4-99.1) \\
n=180 \\
\text { US(3) }\end{array}$ & $\mathrm{Nd}$ \\
\hline PCR & Hologic, SARS-CoV-2 Assay (Panther Fusion System) & ORF1ab & Unk & $\begin{array}{c}98.3(96.8-99.1) \\
n=525 \\
F R, \text { US }(6)\end{array}$ & $\mathrm{Nd}$ \\
\hline PCR & $\begin{array}{l}\text { KH Medical, RADI COVID-19 Detection Kit and RADI } \\
\text { COVID-19 Triple Detection Kit }\end{array}$ & RdRP & Unk & $\begin{array}{c}96.8(89.1-99.1)^{b, c} \\
n=63 \\
\mathrm{CH}, \mathrm{NL}\end{array}$ & $\begin{array}{c}100.0(96.7-100.0) \\
n=112 \\
\mathrm{CH}, \mathrm{NL}\end{array}$ \\
\hline
\end{tabular}

AT: Austria; AU: Australia; BE: Belgium; CH: Switzerland; COVID-19: coronavirus disease; CY: Cyprus; DE: Denmark; E: envelope gene; FI: Finland; FR: France; JP: lapan; N. nucleoprotein gene. Na: not applicable; Nd: not determined, either because there were no data or because there were data from only one country or study; NL: The Netherlands; PL: Poland; S: spike gene; SARS-CoV-2: severe acute respiratory syndrome coronavirus 2; SE: Sweden; SI: Slovenia; UK: United Kingdom; Unk: unknown or unclearly defined; US: United States.

a Positive agreement and specificity values given as value (confidence interval), number of samples $(n=X)$, list of countries (number of studies per country if $>1$ ). Value in bold if both confidence interval width $\leq 5 \%$ and value $\geq 95 \%$ (for positive agreement) or $\geq 98 \%$ (for specificity).

${ }^{b}$ Confidence interval width $>5 \%$.

c Moderate study heterogeneity $(50.0 \leq 12<75.0 \%)$

${ }^{d}$ High study heterogeneity $(\mid 2 \geq 75.0 \%)$.

Rows are sorted alphabetically by test, target and case population. 


\section{TABLE 3B}

Pooled positive agreement and specificity results for SARS-CoV-2 nucleic acid tests with confidence interval width $\leq 5 \%$ for either or both and based on at least two studies, up to 22 August 2020

\begin{tabular}{|c|c|c|c|c|c|}
\hline Category & Test & Target & $\begin{array}{l}\text { Case } \\
\text { population }\end{array}$ & Positive agreement ${ }^{\mathrm{a}}$ & Specificity ${ }^{a}$ \\
\hline PCR & $\begin{array}{l}\text { KH Medical, RADI COVID-19 Detection Kit and } \\
\text { RADI COVID-19 Triple Detection Kit }\end{array}$ & $\mathrm{S}$ & Unk & $\begin{array}{c}98.4(91.5-99.7)^{b} \\
n=63 \\
\mathrm{CH}, \mathrm{NL}\end{array}$ & $\begin{array}{c}100.0(96.7-100.0) \\
\qquad \begin{array}{c}n=112 \\
\mathrm{CH}, \mathrm{NL}\end{array}\end{array}$ \\
\hline PCR & $\begin{array}{c}\text { Primerdesign, genesig Real-Time PCR CoVID- } \\
19 \mathrm{kit}\end{array}$ & RdRP & Unk & $\begin{array}{c}95.3(89.4-98.0)^{b, c} \\
n=106 \\
C H, N L, P L\end{array}$ & $\begin{array}{c}100.0(98.8-100.0) \\
n=307 \\
C H, N L, U K\end{array}$ \\
\hline PCR & R-Biopharm, Ridagene SARS-CoV2 & $\mathrm{E}$ & Unk & $\begin{array}{c}100.0(94.3-100.0)^{b} \\
n=63 \\
\mathrm{CH}, \mathrm{NL}\end{array}$ & $\begin{array}{c}100.0(96.7-100.0) \\
n=112 \\
\mathrm{CH}, \mathrm{NL}\end{array}$ \\
\hline PCR & Roche, COBAS SARS-CoV-2 test & $\begin{array}{l}\text { ORF1ab } \\
\text { or } E\end{array}$ & Unk & $\begin{array}{c}98.8(97.9-99 \cdot 3) \\
n=1,125 \\
\text { AT, CH, DE, FR, SI, US(5) }\end{array}$ & $\begin{array}{c}100.0(90.8- \\
100.0)^{b} \\
n=38 \\
C H, F R\end{array}$ \\
\hline PCR & Seegene, Allplex 2019-nCoV assay & $\mathrm{E}$ & Unk & $\begin{array}{c}85.0(75.6-91.2)^{b, d} \\
n=80 \\
C H, F R, N L\end{array}$ & $\begin{array}{c}100.0(96.7-100.0) \\
n=112 \\
\mathrm{CH}, \mathrm{NL}\end{array}$ \\
\hline PCR & Seegene, Allplex 2019-nCoV assay & RdRP & Unk & $\begin{array}{c}91.3(83.0-95.7)^{b, c} \\
n=80 \\
\mathrm{CH}, \mathrm{FR}, \mathrm{NL}\end{array}$ & $\begin{array}{c}100.0(96.7-100.0) \\
n=112 \\
\mathrm{CH}, \mathrm{NL}\end{array}$ \\
\hline PCR & Tibmolbiol, SARS-CoV (COVID19) E-gene & $\mathrm{E}$ & Unk & $\begin{array}{c}100.0(94.4-100.0)^{b} \\
n=65 \\
C H, U K\end{array}$ & $\begin{array}{c}100.0(98.5-100.0) \\
n=250 \\
C H, U K\end{array}$ \\
\hline
\end{tabular}

AT: Austria; AU: Australia; BE: Belgium; CH: Switzerland; COVID-19: coronavirus disease; CY: Cyprus; DE: Denmark; E: envelope gene; FI: Finland; FR: France; JP: Japan; N: nucleoprotein gene; Na: not applicable; Nd: not determined, either because there were no data or because there were data from only one country or study; NL: The Netherlands; PL: Poland; S: spike gene; SARS-CoV-2: severe acute respiratory syndrome coronavirus 2; SE: Sweden; SI: Slovenia; UK: United King, Nom; Unk: unknown or unclearly defined; US. United States.

a Positive agreement and specificity values given as value (confidence interval), number of samples $(n=X)$, list of countries (number of studies per country if $>1)$. Value in bold if both confidence interval width $\leq 5 \%$ and value $\geq 95 \%$ (for positive agreement) or $\geq 98 \%$ (for specificity).

${ }^{\mathrm{b}}$ Confidence interval width $>5 \%$.

${ }^{c}$ Moderate study heterogeneity $(50.0 \leq 12<75.0 \%)$.

${ }^{d}$ High study heterogeneity $(\mid 2 \geq 75.0 \%)$.

Rows are sorted alphabetically by test, target and case population. 


\section{FIGURE 2}

Independently assessed vs manufacturer-reported clinical sensitivity and specificity per SARS-CoV-2 test, up to 22 August $2020(\mathrm{n}=55)$

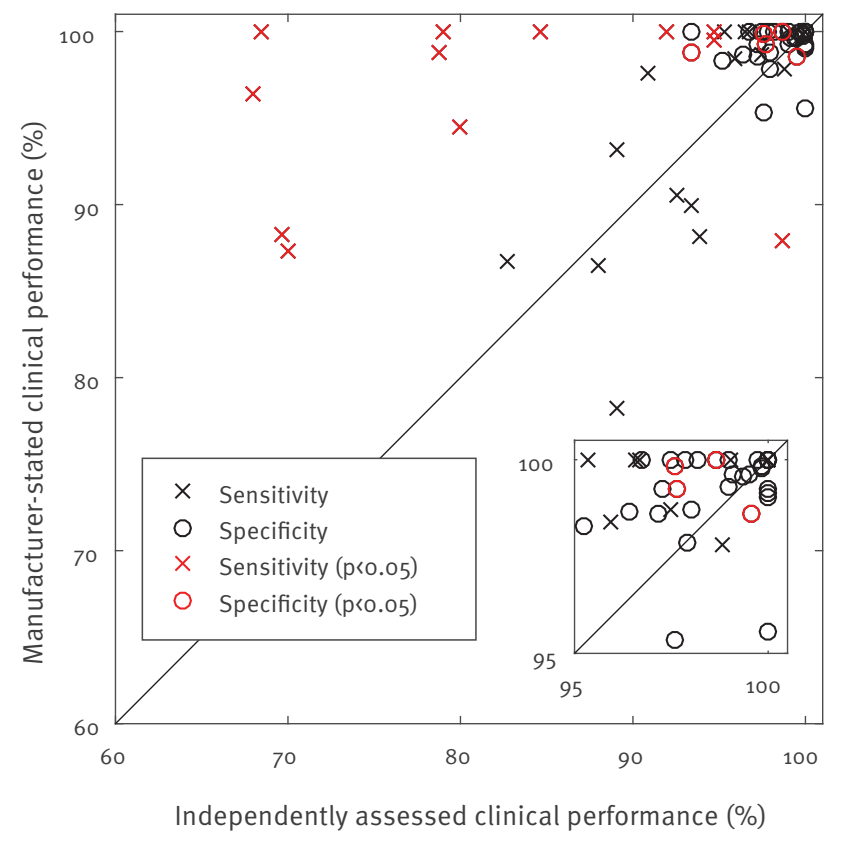

SARS-CoV-2: severe acute respiratory syndrome coronavirus 2.

Significantly different $(p<0.05)$ results are highlighted. Independently assessed results limited to those with $95 \%$ confidence interval width $\leq 5 \%$. The inset expands the $95-100 \%$ region.

have a substantial effect on performance. These are often not specified in detail or several processes may be allowed according to the instructions for use, which can have contributed to study heterogeneity. While this review addresses a pressing need for actionable clinical performance data, ideally, the clinical performance should be assessed through prospective studies or clinical trials with a guaranteed unbiased sample selection for a clearly defined target population and intended use of the test. Given the difficulty of assessing and extracting the data from individual studies in a coherent way, we recommend that the Standard for Reporting of Diagnostic Accuracy Studies (STARD) should also be followed when publishing the results [27].

In this context, the selection of the reference test is particularly important with respect to reference negative samples. As described in some of the assessed studies, it should be avoided that index test results are considered as false positives while the samples are from actual cases; for this reason we excluded nucleic acid-negative samples from suspected COVID19 patients altogether. We therefore expect little bias in the specificity results, except potentially from underor overrepresentation of confounders. This is especially relevant for seroprevalence studies where, in a lowprevalence situation, in particular the specificity of the test needs to be well defined and high. On the other hand, sensitivity results using a nucleic acid test as reference should be interpreted with caution because the positive samples may exclude some actual cases.

Possibilities to improve the reference test can include testing - potentially only the false positives - with a second reference nucleic acid test preferably targeting different genes, testing more than one sample from the same patient including for antibodies at a later time point, testing samples from both upper and lower respiratory tracts, and sequencing the sample. The handling of intermediate index test results is an issue that needs to be described in studies and in general, these should be considered as positive results rather than as negatives or excluding them from the validation, since in clinical practice they would normally require further follow-up to confirm the positivity of the sample. Finally, the quality of the execution of the tests is also an important factor. For non-point-of-care tests, external quality assessment exercises using well validated standard reference materials remain a critical tool to detect and address such issues.

\section{Conclusion}

Given the study limitations, the authors and organisations contributing to this study in no way recommend the use of the listed commercial tests over other not listed commercial or in-house tests. The clinical performance of tests may also change over time as the virus population evolves. We recommend, however, continuous monitoring of clinical performance both in Europe and globally, which is key for reliable monitoring of the pandemic and which will also support vaccine and antiviral development. These results should be shared publicly in a timely manner.

European COVID-19 microbiological laboratories group Marjan Van Esbroeck: Institute of Tropical Medicine, Antwerpen, Belgium

Pieter Vermeersch: Clinical Department of Laboratory Medicine and National Reference Center for Respiratory Pathogens, University Hospitals Leuven, Leuven, Belgium

Kurt Beuselinck: Clinical Department of Laboratory Medicine and National Reference Center for Respiratory Pathogens, University Hospitals Leuven, Leuven, Belgium

\section{Christos Karagiannis: Nicosia General Hospital, Cyprus}

Merit Melin: Department of Health Protection, Expert Microbiology Unit, Finnish Institute for Health and Welfare (THL), Helsinki, Finland

Nina Ekström: Department of Health Protection, Expert Microbiology Unit, Finnish Institute for Health and Welfare (THL), Helsinki, Finland

Iris Erlund: Department of Government Services, Finnish Institute for Health and Welfare (THL), Helsinki, Finland

Terhi Vihervaara: Department of Government Services, Finnish Institute for Health and Welfare (THL), Helsinki, Finland 
Vanessa Escuret: Laboratoire de Virologie des HCL, Institut des Agents Infectieux, CNR des virus à transmission respiratoire (dont la grippe), Groupement Hospitalier Nord, Lyon, France

Emilie Frobert: Laboratoire de Virologie des HCL, Institut des Agents Infectieux, CNR des virus à transmission respiratoire (dont la grippe), Groupement Hospitalier Nord, Lyon, France

Alexandre Gaymard: Laboratoire de Virologie des $\mathrm{HCL}$, Institut des Agents Infectieux, CNR des virus à transmission respiratoire (dont la grippe), Groupement Hospitalier Nord, Lyon, France

\section{Andreas Mentis: Hellenic Pasteur Institute, Athens, Greece}

Stavroula Lampropoulou: Hellenic Pasteur Institute, Athens, Greece

Ivan-Christian Kurolt: Research unit, University Hospital for Infectious Diseases “Dr. Fran Mihaljević”, Zagreb, Croatia

Tamir Abdelrahman: Department of Microbiology, Laboratoire national de santé, Luxembourg

Trung Nguyen: Department of Microbiology, Laboratoire national de santé, Luxembourg

Guillaume Fournier: Department of Microbiology, Laboratoire national de santé, Luxembourg

Chantal B.E.M. Reusken: Centre for Infectious Disease Control, National Institute for Public Health and the Environment, The Netherlands

Maaike J.C. van den Beld: Centre for Infectious Disease Control, National Institute for Public Health and the Environment, The Netherlands

Janette Rahamat-Langendoen MD PhD: Department of Medical Microbiology, Radboud University Medical Center, Nijmegen

Marjolijn C.A. Wegdam-Blans: Department of Medical Microbiology, PAMM, Veldhoven, The Netherlands

Jeroen H. T. Tjhie: Department of Medical Microbiology, PAMM, Veldhoven, The Netherlands

Peter Croughs: Department of Medical Microbiology and Infectious Diseases, Erasmus Medical Center, Rotterdam, The Netherlands

Corine H. GeurtsvanKessel: Department of Virology, Erasmus Medical Center, Rotterdam, The Netherlands

Johan Reimerink: Centre for Infectious Diseases Research, Diagnostics and Laboratory Surveillance, Centre for Infectious Disease Control, National Institute for Public Health and the Environment, The Netherlands

David S.Y. Ong: Department of Medical Microbiology and Infection Control, Franciscus Gasthuis \& Vlietland, Rotterdam, The Netherlands, Department of Epidemiology, Julius Center for Health Sciences and Primary Care, University Medical Center Utrecht, Utrecht, The Netherlands

Hans G.M. Koeleman: Department of Medical Microbitherlands

Hannke Berkhout: Canisius-Wilhelmina hospital, Nijmegen, The Netherlands
Christel F.M. van der Donk: Canisius-Wilhelmina hospital, Nijmegen, The Netherlands

Menno D. de Jong MD PhD: Department of Medical Microbiology \& Infection prevention, Amsterdam University Medical Centers, The Netherlands

Rens Zonneveld MD PhD: Department of Medical Microbiology, Amsterdam University Medical Center, Amsterdam, The Netherlands

Suzanne Jurriaans PhD: Department of Medical Microbiology, Amsterdam University Medical Center, Amsterdam, The Netherlands

Nathalie Van Burgel: Hagaziekenhuis, The Hague, The Netherlands

Bas B. Wintermans MD: Department of Medical Microbiology and Immunology, Admiraal de Ruyter Hospital, Vlissingen, The Netherlands

Ger T. Rijkers: Department of Medical Microbiology and Immunology, Admiraal de Ruyter Hospital, Goes, The Netherlands, Elisabeth-Tweesteden Hospital, Tilburg, The Netherlands

Jean-Luc Murk MD PhD: Elisabeth-Tweesteden Hospital, Tilburg, The Netherlands

Khoa T.D. Thai MD PhD: Unit of Medical Microbiology, Starshl Medical Diagnostic Center, Rotterdam, The Netherlands, Department of Medical Microbiology and Infectious Diseases, Erasmus Medical Center, Rotterdam, The Netherlands

Melanie J de Graaf: Department of Medical Microbiology, University Medical Centre, Utrecht, The Netherlands, Saltro Diagnostic Centre, Utrecht, The Netherlands

Annemarie van 't Veen: Department of Medical Microbiology, University Medical Centre, Utrecht, The Netherlands, Saltro Diagnostic Centre, Utrecht, The Netherlands

Cornelis P. Timmerman: Central Bacteriology and Serology Laboratory, Tergooi Hospital, Hilversum, The Netherlands

Annette van Corteveen-Splinter: Central Bacteriology and Serology Laboratory, Tergooi Hospital, Hilversum, The Netherlands

Felix Geeraedts: Laboratory for Medical Microbiology and Public Health, Hengelo, The Netherlands

Adrian Klak: Laboratory for Medical Microbiology and Public Health, Hengelo, The Netherlands

Maria M. Konstantinovski MD: Reinier Haga Medical Diagnostic Centre, Delft, The Nederlands

Manou R. Batstra: Reinier Haga Medical Diagnostic Centre, Delft, The Nederlands

K. A. Heemstra MD PhD: Alrijne Zorggroep, Leiderdorp, The Netherlands

Jos J. Kerremans MD PhD: Alrijne Zorggroep, Leiderdorp, The Netherlands

Inge H. M. van Loo: Department of Medical Microbiology, Maastricht University Medical Center, The Netherlands, Care and Public Health Research Institute, Maastricht University 
Paul H. M. Savelkoul: Department of Medical Microbiology, Maastricht University Medical Center, The Netherlands, Care and Public Health Research Institute, Maastricht University

Johan Kissing: Department of Medical Microbiology and Infection prevention, Gelre Hospitals, Apeldoorn, The Netherlands

Paul Martijn den Reijer: Department of Medical Microbiology and Infection prevention, Gelre Hospitals, Apeldoorn, The Netherlands

Anne Russcher: Department of Medical Microbiology, Medical Meander Center, Amersfoort, The Netherlands

Moniek Heusinkveld PhD: Department of Medical Microbiology, Hospital Gelderse Vallei, Ede, The Netherlands

Ellen van Lochem: Department of Medical Microbiology and Immunology, Hospital Rijnstate, The Netherlands

Steven F. T. Thijsen: Medical Microbiology and Immunology, Diakonessen Hospital, Utrecht, The Netherlands

Michiel Heron: Medical Microbiology and Immunology, Diakonessen Hospital, Utrecht, The Netherlands

Susanne P. Stoof MD PhD: Department of Medical Microbiology, Comicro, Hoorn, The Netherlands

Sim van Gyseghem BSc: Department of Medical Microbiology, Comicro, Hoorn, The Netherlands

Sylvia B. Debast MD PhD: Laboratory of Clinical Microbiology and Infectious Diseases, Isala Hospital, Zwolle, The Netherlands

Claudy Oliveira dos Santos MD: Laboratory of Clinical Microbiology and Infectious Diseases, Isala Hospital, Zwolle, The Netherlands

Bjorn L. Herpers MD PhD: Regional Public Health Laboratory Kennemerland, The Netherlands

Theo Mank PhD: Regional Public Health Laboratory Kennemerland, The Netherlands

Kin Ki Jim: Department of Medical Microbiology and Infection Control, Jeroen Bosch Hospital, 's-Hertogenbosch, The Netherlands, Department of Medical Microbiology and Infection Prevention, Amsterdam University Medical Centers, Amsterdam institute for Infection and Immunity, Amsterdam, The Netherlands

Peter C. Wever: Department of Medical Microbiology and Infection Control, Jeroen Bosch Hospital, 's-Hertogenbosch, The Netherlands

Jutte J.C. de Vries: Department of Medical Microbiology, Leiden University Medical Center, Leiden, The Netherlands

Martine Hoogewerf: Department of Medical Microbiology, Northwest Hospital Group, Alkmaar, The Netherlands

Deborah J. Kaersenhout MD MSc: Atalmedial Medical Microbiology Laboratory, Amsterdam, the Netherlands

Annette M. Stemerding: Deventer Ziekenhuis, Deventer, the Netherlands

Babette C. van Hees: Deventer Ziekenhuis, Deventer, the Netherlands
Vishal Hira: Department of Medical Microbiology and Infection Prevention, Groene Hart Ziekenhuis, Gouda, the Netherlands

Anne E. Bos: Department of Medical Microbiology and Infection Prevention, Groene Hart Ziekenhuis, Gouda, the Netherlands

Leontine Mulder: Clinical Laboratory, Medlon B.V., Enschede, The Netherlands

Michiel van Rijn MD: Medical Laboratory, Ikazia Hospital, Rotterdam, The Netherlands

Aleksander Michalski: 1st Clinical Military Hospital with Outpatient Clinic, Lublin, Poland

Marta Pakieta: Voivodeship Sanitary Epidemiological Station, Warsaw, Poland

Anna Siewierska-Puchlerska: Voivodeship Sanitary Epidemiological Station, Warsaw, Poland

Jarostaw Paciorek: Voivodeship Sanitary Epidemiological Station, Warsaw, Poland

Ewa Gajda: Epidemiological Response Centre of The Polish Armed Forces, Warsaw, Poland

Katarzyna Pancer PhD: Department of Virology, BSL3 Laboratory, COVID-19 NIPH-NIH-NRI team, National Institute of Public Health-National Institute of Hygiene - National Research Institute, Warsaw, Poland

Agnieszka Kołakowska-Kulesza: Department of Virology, COVID-19 NIPH-NIH-NRI team, National Institute of Public Health-National Institute of Hygiene - National Research Institute, Warsaw, Poland

Magdalena Nowakowska: Department of Bacteriology and Biocontamination Control, COVID-19 NIPH-NIH-NRI team, National Institute of Public Health-National Institute of Hygiene - National Research Institute, Warsaw, Poland

Raquel Guiomar: Instituto Nacional de Saúde Dr. Ricardo Jorge, I.P., Portugal.

Líbia Zé-Zé: Instituto Nacional de Saúde Dr. Ricardo Jorge, I.P., Portugal.

Inês Costa: Instituto Nacional de Saúde Dr. Ricardo Jorge, I.P., Portugal.

Johan Brynedal Öckinger: Department of Virology, Clinical Microbiology, Karolinska University Laboratory, Karolinska University Hospital, Stockholm, Sweden

Berit Hammas: Department of Virology, Clinical Microbiology, Karolinska University Laboratory, Karolinska University Hospital, Stockholm, Sweden

Katarina Prosenc: National Laboratory for Health, Environment and Food Slovenia, Laboratory for Public Health Virology

Nataša Berginc: National Laboratory for Health, Environment and Food Slovenia, Laboratory for Public Health Virology

\section{Acknowledgements}

We would like to acknowledge the technicians in the European microbiology laboratories who work hard to support the 
control of COVID-19 and supported the validations described in this manuscript.

We would like to acknowledge the companies that made some of the kits available for evaluation to some of the laboratories.

Funding: Pieter Vermeersch is a senior clinical investigator of the FWO-Vlaanderen. (Note: FWO-Vlaanderen is the Flemish public Fund for Scientific Research.)

\section{Conflict of interest}

None declared.

\section{Authors' contributions}

Ivo Van Walle: conceptualisation, methodology, data curation, formal analysis, writing-review, editing

Katrin Leitmeyer: conceptualisation, methodology, data curation, writing-review, editing

Eeva K. Broberg: conceptualisation, methodology, data curation, writing-review, editing.

European COVID-19 microbiological laboratories group:

Marjan Van Esbroeck: investigation, data curation, writing-review

Pieter Vermeersch: data curation, writing-review, editing

Kurt Beuselinck: data curation

Christos Karagiannis: investigation, data curation, writing-review

Merit Melin: conceptualisation, investigation, data curation, writing-review

Nina Ekström: investigation, data curation

Iris Erlund: methodology

Terhi Vihervaara: methodology

Vanessa Escuret: investigation, data curation, writing-review Emilie Frobert: investigation, data curation, writing-review

Alexandre Gaymard: investigation, data curation, writing-review

Andreas Mentis: methodology, data analysis

Stavroula Lampropoulou: investigation, data curation

Ivan-Christian Kurolt: investigation, data curation, writing-review

Tamir Abdelrahman: investigation, data curation, writing-review

Trung Nguyen: investigation, data curation, writing-review

Guillaume Fournier: investigation, data curation, writing-review

Chantal B.E.M. Reusken: conceptualisation, investigation, data curation, methodology, writing-review
Maaike J.C. van den Beld: investigation, data curation, methodology, writing-review

Janette Rahamat-Langendoen MD PhD: investigation, data curation, writing-review

Marjolijn C.A. Wegdam-Blans: investigation, data curation, writing-review

Jeroen H. T. Tjhie: investigation, data curation, writing-review Peter Croughs: investigation, data curation, writing-review

Corine H. GeurtsvanKessel: investigation, data curation, writing-review

Johan Reimerink: Investigation, data curation

David S.Y. Ong: investigation, data curation, writing-review

Hans G.M. Koeleman: investigation, data curation, writing-review

Hannke Berkhout: investigation, data curation, writing-review

Christel F.M. van der Donk: investigation, data curation, writing-review

Menno D. de Jong MD PhD: investigation, data curation, writing-review

Rens Zonneveld MD PhD: investigation, data curation, writing-review

Suzanne Jurriaans PhD: investigation, data curation, writing-review

Nathalie Van Burgel: investigation, data curation, writing-review

Bas B. Wintermans MD: investigation, data curation, writing-review

Ger T. Rijkers: investigation, data curation, writing-review

Jean-Luc Murk MD PhD: investigation, data curation, writing-review

Khoa T.D. Thai MD PhD: investigation, data curation, writing-review

Melanie J de Graaf: investigation, data curation, writing-review

Annemarie van 't Veen: investigation, data curation, writing-review

Cornelis P. Timmerman: investigation, data curation, writing-review

Annette van Corteveen-Splinter: investigation, data curation, writing-review

Felix Geeraedts: investigation, data curation, writing-review Adrian Klak: investigation, data curation, writing-review

Maria M. Konstantinovski MD: investigation, data curation, writing-review

Manou R. Batstra: investigation, data curation, writing-review

K. A. Heemstra: investigation, data curation 
Jos J. Kerremans: investigation, data curation, writing-review Inge H. M. van Loo: investigation, data curation, writing-review

Paul H. M. Savelkoul: investigation, data curation

Johan Kissing: investigation, data curation

Paul Martijn den Reijer: investigation, data curation, writing-review

Anne Russcher: investigation, data curation, writing-review

Moniek Heusinkveld PhD: investigation, data curation, writing-review

Ellen van Lochem: investigation, data curation, writing-review

Steven F. T. Thijsen: investigation, data curation, writing-review

Michiel Heron: investigation, data curation, writing-review

Susanne P. Stoof MD PhD: investigation, data curation, writing-review

Sim van Gyseghem BSc: investigation, data curation, writing-review

Sylvia B. Debast MD PhD: investigation, data curation, writing-review

Claudy Oliveira dos Santos MD: investigation, data curation, writing-review

Bjorn L. Herpers MD PhD: investigation, data curation, writing-review

Theo Mank PhD: investigation, data curation, writing-review

Kin Ki Jim: investigation, data curation, writing-review

Peter C. Wever: investigation, data curation, writing-review

Jutte J.C. de Vries: investigation, data curation, writing-review

Martine Hoogewerf: investigation, data curation, writing-review

Deborah J. Kaersenhout MD MSc: data curation, writing-review

Annette M. Stemerding: investigation, data curation, writing-review

Babette C. van Hees: investigation, data curation, writing-review

Vishal Hira: investigation, data curation, writing-review

Anne E. Bos: investigation, data curation, writing-review

Leontine Mulder: investigation, data curation, writing-review

Michiel van Rijn MD: investigation, data curation, writing-review

Aleksander Michalski: investigation, data curation, writing-review

\section{Marta Pakieta: writing-review}

Anna Siewierska-Puchlerska: investigation, data curation
Jarosław Paciorek: investigation, data curation

Ewa Gajda: investigation, data curation

Katarzyna Pancer: investigation, data curation, writing-review

Agnieszka Kotakowska-Kulesza: investigation, data curation Magdalena Nowakowska: investigation, data curation Raquel Guiomar: writing-review

Líbia Zé-Zé: data curation, writing-review Inês Costa: investigation, data curation, writing-review Johan Brynedal Öckinger: investigation, data curation, writing-review

Berit Hammas: investigation, data curation, writing-review

Katarina Prosenc: investigation, data curation

Nataša Berginc: investigation, data curation

\section{References}

1. United States Food and Drug Administration (FDA). Emergency use authorizations for medical devices. Silver Spring: FDA.

[Accessed: 20 Jul 2020]. Available from: https://www.fda. gov/medical-devices/emergency-situations-medical-devices/ emergency-use-authorizations-medical-devices\#covid19ivd

2. World Health Organisation (WHO). WHO Emergency Use Listing for In vitro diagnostics (IVDs) Detecting SARS-CoV-2. Geneva: WHO. [Accessed: 31 Oct 2021]. Available from: https://www.who.int/publications/m/ item/200922-eul-sars-cov2-product-list

3. Joint Research Centre of the European Commission. COVID-19 in vitro diagnostic devices and test methods database. Brussels: European Commission. [Accessed: 20 Jul 2020]. Available from: https://covid-19-diagnostics.jrc.ec.europa.eu

4. Foundation for Innovative Diagnostics (FIND). COVID-19 tests (commercialized \& in development). Geneva: FIND. [Accessed: 20 Jul 2020]. Available from: https://www.finddx.org/covid-19/ tests

5. United States Food and Drug Administration (FDA). openFDA COVID-19 serological testing evaluations. Silver Spring: FDA. [Accessed: 20 Jul 2020]. Available from: https://open.fda.gov/ apis/device/covid19serology/download

6. Deeks JJ, Dinnes J, Takwoingi Y, Davenport C, Spijker R, TaylorPhillips S, et al. Antibody tests for identification of current and past infection with SARS-CoV-2. Cochrane Database Syst Rev. 2020;6:CD013652. https://doi.org/10.1002/14651858. CD013652 PMID: 32584464

7. European Network for Health Technology Assessment (EUnetHTA). Rapid collaborative review on the current role of antibody tests for novel coronavirus SARS-CoV-2 in the management of the pandemic. Diemen: EUnetHTA; 2020. Available from: https://www.eunethta.eu/wp-content/ uploads/2020/06/RCR_0T_01-_Antibody-tests-for-SARSCoV-2_23-06-2020.pdf

8. Lisboa Bastos M, Tavaziva G, Abidi SK, Campbell JR, Haraoui LP, Johnston JC, et al. Diagnostic accuracy of serological tests for covid-19: systematic review and meta-analysis. BMJ. 2020;370:m2516. https://doi.org/10.1136/bmj.m2516 PMID: 32611558

9. Dinnes J, Deeks JJ, Adriano A, Berhane S, Davenport C, Dittrich $S$, et al. Rapid, point-of-care antigen and molecular-based tests for diagnosis of SARS-CoV-2 infection. Cochrane Database Syst Rev. 2020;8(8):CD013705. PMID: 32845525

10. Attwood LO, Francis MJ, Hamblin J, Korman TM, Druce J, Graham M. Clinical evaluation of AusDiagnostics SARS-CoV-2 multiplex tandem PCR assay. J Clin Virol. 2020;128:104448. https://doi.org/10.1016/j.jcv.2020.104448 PMID: 32460173

11. Pan Y, Li X, Yang G, Fan J, Tang Y, Zhao J, et al. Serological immunochromatographic approach in diagnosis with SARSCoV-2 infected COVID-19 patients. J Infect. 2020;81(1):e28-32. https://doi.org/10.1016/j.jinf.2020.03.051 PMID: 32283141 
12. Wang P. Combination of serological total antibody and RT-PCR test for detection of SARS-COV-2 infections. J Virol Methods. 2020;283:113919. https://doi.org/10.1016/j. jviromet.2020.113919 PMID: 32554043

13. Zhao J, Yuan Q, Wang H, Liu W, Liao X, Su Y, et al. Antibody responses to SARS-CoV-2 in patients of novel coronavirus disease 2019. Clin Infect Dis. 2020;71(16):2027-34. https://doi. org/10.1093/cid/ciaa344 PMID: 32221519

14. Zou L, Ruan F, Huang M, Liang L, Huang H, Hong Z, et al. SARSCoV-2 viral load in upper respiratory specimens of infected patients. N Engl J Med. 2020;382(12):1177-9. https://doi. org/10.1056/NEJMc2001737 PMID: 32074444

15. Green DA, Zucker J, Westblade LF, Whittier S, Rennert H, Velu P, et al. Clinical performance of SARS-CoV-2 molecular tests. J Clin Microbiol. 2020;58(8):e00995-20. https://doi. org/10.1128/JCM.00995-20 PMID: 32513858

16. Kim H, Hong H, Yoon SH. Diagnostic performance of CT and reverse transcriptase polymerase chain reaction for coronavirus disease 2019: a meta-analysis. Radiology. 2020;296(3):E145-55. https://doi.org/10.1148/ radiol.2020201343 PMID: 32301646

17. World Health Organization (WHO). Laboratory testing for coronavirus disease (COVID-19) in suspected human cases: interim guidance, 19 March 2020. Geneva: WHO; 2020. Available from: https://apps.who.int/iris/handle/10665/331501

18. Viechtbauer W. Conducting meta-analyses in $\mathrm{R}$ with the metafor package. J Stat Softw. 2010;36(3):1-48. https://doi. org/10.18637/jss.v036.io3

19. World Health Organization (WHO). COVID-19 target product profiles for priority diagnostics to support response to the COVID-19 pandemic v.0.1. Geneva: WHO; 2020. Available from: https://www.who.int/publications/m/item/covid-19-targetproduct-profiles-for-priority-diagnostics-to-support-responseto-the-covid-19-pandemic-v.0.1

20. Sethuraman N, Jeremiah SS, Ryo A. Interpreting diagnostic tests for SARS-CoV-2. JAMA. 2020;323(22):2249-51. https:// doi.org/10.1001/jama.2020.8259 PMID: 32374370

21. La Marca A, Capuzzo M, Paglia T, Roli L, Trenti T, Nelson SM. Testing for SARS-CoV-2 (COVID-19): a systematic review and clinical guide to molecular and serological in-vitro diagnostic assays. Reprod Biomed Online. 2020;41(3):483-99. https:// doi.org/10.1016/j.rbmo.2020.06.001 PMID: 32651106

22. Böger B, Fachi MM, Vilhena RO, Cobre AF, Tonin FS, Pontarolo $R$. Systematic review with meta-analysis of the accuracy of diagnostic tests for COVID-19. Am J Infect Control. 2021;49(1):21-9. https://doi.org/10.1016/j.ajic.2020.07.011 PMID: 32659413

23. Caini S, Bellerba F, Corso F, Díaz-Basabe A, Natoli G, Paget J, et al. Meta-analysis of diagnostic performance of serological tests for SARS-CoV-2 antibodies up to 25 April 2020 and public health implications. Euro Surveill. 2020;25(23):2000980. https://doi.org/10.2807/1560-7917.ES.2020.25.23.2000980 PMID: 32553061

24. Moura DTH, McCarty TR, Ribeiro IB, Funari MP, Oliveira PVAG, Miranda Neto AA, et al. Diagnostic characteristics of serological-based COVID-19 testing: a systematic review and meta-analysis. Clinics (São Paulo). 2020;75:e2212. https://doi. org/10.6061/clinics/2020/e2212 PMID: 32785570

25. Vermeersch $P$, André E. How the European in vitro diagnostic regulation could negatively impact the European response to the next pandemic: an urgent call for action before May 2022. Clin Microbiol Infect. 2021;27(8):1074-5. https://doi. org/10.1016/j.cmi.2021.05.009 PMID: 33979703

26. European Commission (EC). Questions and answers on the progressive roll-out of the new In Vitro Diagnostic Medical Devices Regulation. Brussels: EC. [Accessed: 1 Nov 2021]. Available from: https://ec.europa.eu/commission/presscorner/ detail/en/qanda_21_5210

27. Cohen JF, Korevaar DA, Altman DG, Bruns DE, Gatsonis CA, Hooft L, et al. STARD 2015 guidelines for reporting diagnostic accuracy studies: explanation and elaboration. BMJ Open. 2016;6(11):e012799. https://doi.org/10.1136/ bmjopen-2016-012799 PMID: 28137831

\section{License, supplementary material and copyright}

This is an open-access article distributed under the terms of the Creative Commons Attribution (CC BY 4.0) Licence. You may share and adapt the material, but must give appropriate credit to the source, provide a link to the licence and indicate if changes were made.
Any supplementary material referenced in the article can be found in the online version.

This article is copyright of the authors or their affiliated institutions, 2021. 